\title{
Using Epidemiological Principles to Explain Fungicide Resistance Management Tactics: Why do Mixtures Outperform Alternations?
}

\author{
James A. D. Elderfield, Francisco J. Lopez-Ruiz, Frank van den Bosch, and Nik J. Cunniffe†
}

First and fourth authors: Department of Plant Sciences, University of Cambridge, Cambridge, CB2 3EA, United Kingdom; second author: Curtin University, Centre for Crop and Disease Management, Department of Environment and Agriculture, Bentley, WA 6845, Australia; and third author: Rothamsted Research, Harpenden, AL5 2JQ, United Kingdom.

Accepted for publication 25 January 2018.

\begin{abstract}
Whether fungicide resistance management is optimized by spraying chemicals with different modes of action as a mixture (i.e., simultaneously) or in alternation (i.e., sequentially) has been studied by experimenters and modelers for decades. However, results have been inconclusive. We use previously parameterized and validated mathematical models of wheat Septoria leaf blotch and grapevine powdery mildew to test which tactic provides better resistance management, using the total yield before resistance causes disease control to become economically ineffective ("lifetime yield") to measure effectiveness. We focus on tactics involving the combination of a lowrisk and a high-risk fungicide, and the case in which resistance to the high-risk chemical is complete (i.e., in which there is no partial resistance). Lifetime

yield is then optimized by spraying as much low-risk fungicide as is permitted, combined with slightly more high-risk fungicide than needed for acceptable initial disease control, applying these fungicides as a mixture. That mixture rather than alternation gives better performance is invariant to model parameterization and structure, as well as the pathosystem in question. However, if comparison focuses on other metrics, e.g., lifetime yield at full label dose, either mixture or alternation can be optimal. Our work shows how epidemiological principles can explain the evolution of fungicide resistance, and also highlights a theoretical framework to address the question of whether mixture or alternation provides better resistance management. It also demonstrates that precisely how spray tactics are compared must be given careful consideration.
\end{abstract}

Designing long-lasting, effective tactics to control plant disease remains a key challenge (Cunniffe et al. 2015). Fungicide resistance management —optimizing deployment to delay emergence or spread of resistant pathogen strains - has been studied for decades (Russell 2005). Many tactics have been proposed. For a single fungicide, resistance management can be based on the method of application, changing the dose (van den Bosch et al. 2011), the timing (van den Berg et al. 2013), whether treatment is applied to the leaves or on the seed (Kitchen et al. 2016), the spatial pattern of spraying (Parnell et al. 2006), or the number of sprays per season (van den Berg et al. 2016). However, for disease control as well as resistance management, fungicides with different modes of action are very often combined in a spray program (van den Bosch et al. 2014b).

Significant attention has therefore been devoted to how to best combine fungicide applications. Possibilities include a mixture, spraying the two fungicides at the same time, or as an alternation, applying sequentially. The risk of resistance development varies between fungicides (Brent and Hollomon 2007). Resistance emerges to some chemicals within a few years of use, while others provide durable control for decades. We distinguish high-risk fungicides, to which resistance is already present or very likely to emerge, and low-risk fungicides, to which no significant resistance has yet been observed. We focus here on the case of mixture and alternation of a single high-risk fungicide with a single low-risk when there is a fungal strain fully resistant to the high-risk. Despite many experimental (Cooke et al. 2004; Dovas et al. 1976; Lamondia 2001; Sanders et al. 1985; Vali and Moorman 1992) and modeling

†Corresponding author: N. J. Cunniffe; E-mail: njc1001@cam.ac.uk

*The $\boldsymbol{e}$-Xtra logo stands for "electronic extra" and indicates that one supplementary figure, two supplementary tables, and one supplementary text file are published online.

Copyright $\odot 2018$ The Author(s). This is an open access article distributed under the CC BY 4.0 International license.
(Birch and Shaw 1997; Doster et al. 1990; Hobbelen et al. 2011a, 2013; Josepovits 1989; Josepovits and Dobrovolszky 1985; Kable and Jeffery 1980; Shaw 1989a; Skylakakis 1981) studies focusing on precisely this situation, no conclusive answer has emerged to the important but very simple question: does mixture or alternation provide better resistance management?

Although previous studies have led to equivocal results, fungicide mixtures have often been found to provide superior resistance management (van den Bosch et al. 2014b). van den Bosch et al. (2014a) introduced a simple set of governing principles as a theoretical framework to synthesize these and other results concerning resistance management, formalizing previous concepts from the literature (Milgroom and Fry 1988; Staub and Sozzi 1983). These governing principles are based on constant rates of selection for resistance. We generalize this here, quantifying total selection for resistance by integrating a time-varying selection coefficient over time. The selection coefficient is defined as the difference in fitness between fungicide-sensitive and fungicide-resistant strains

$$
s=r_{R}-r_{S}
$$

where $r_{R}$ and $r_{S}$ are the per capita growth rates of the resistant and sensitive pathogen strains, respectively. The total amount of selection for resistance is then given by the cumulative selection coefficient

$$
\sigma=\int_{0}^{T} s(t) \mathrm{d} t
$$

in which $T$ is the time of exposure to fungicide. Selection for resistance can therefore be reduced by decreasing both $r_{R}$ and $r_{S}$, by decreasing $r_{R}$ only, or by decreasing $T$ (van den Bosch et al. 2014a).

The governing principles can be applied to the comparison between mixture and alternation. Fungicide mixtures can reduce 
selection since the low-risk mixing partner suppresses growth rates of both sensitive and resistant pathogen strains. Mixtures may also permit the use of less high-risk fungicides, decreasing differences in growth rate between strains. However, due to the concave shape of fungicide dose-response curves, mixtures experience a selective cost from "dose-splitting". Splitting a dose of fungicide over multiple sprays increases the total effect on the pathogen and thus the selection pressure imposed (van den Bosch et al. 2014a). Additionally, alternations tend to reduce selection by reducing the number of sprays of high-risk and thus the time of exposure. Our work here assesses in detail, for the first time, the impact of this trade-off between suppression from the mixing partner and dose-splitting.

The structure of a mathematical model affects the conclusions to which it leads (Cunniffe et al. 2012). Older models tended to collapse epidemics into exponential growth of fungicide-sensitive and fungicide-resistant strains (Kable and Jeffery 1980; Shaw 1989b; Skylakakis 1981), with more realistic, compartmental-type models only appearing later (Gubbins and Gilligan 1999; Hall et al. 2004; Mikaberidze et al. 2014, 2017; Parnell et al. 2005, 2006). The current vogue emphasizes detailed, system-specific models (Kitchen et al. 2016; van den Berg et al. 2016). Here we test explicitly how model structure affects our results. We also test the effect of pathosystem by performing our analyses for two systems. Most of our results are based on a model of Septoria leaf blotch (Zymoseptoria tritici) on winter wheat, but we test robustness via a model of powdery mildew (Erysiphe necator) on grapevine.

We use these models to compare alternation and mixtures of lowand high-risk fungicides. We address the following questions.

1. Is it better to apply two fungicides as a mixture, or as an alternation?

2. How does this depend on fungicide dose, and the level of disease control?

3. How can an optimal dose and spray program be determined?

4. Are results conditioned on (i) values of parameters governing epidemiological rates and fungicide performance; (ii) model structure; and (iii) the pathosystem under consideration?

\section{MATERIALS AND METHODS}

Modeling fungicide. Application tactics. Our focus here is to compare mixtures (in which both chemicals are applied in each spray) with alternation (in which the chemical used alternates between sprays). However, for alternation this introduces a dependence upon which chemical is sprayed first in any given growing season. We therefore compare a total of three tactics.

1. Mixture. Both the high- and low-risk fungicide are applied at each spray.

2. Alternation High-Low. Alternate sprays of high- and lowrisk, with high-risk sprayed first in each season.

3. Alternation Low-High. High- and low-risk alternate, with low-risk first.

Since each fungicide is sprayed twice as often when part of a mixture, we halve the dose to conserve the total amount of each chemical applied per season (van den Bosch et al. 2014a).

Fungicide dynamics. Concentrations of both fungicides are set to zero at the start of each season. The concentration of a fungicide is sharply increased whenever it is sprayed, with the timing depending on the pathosystem. Different fungicides decay at different rates in our model, although between sprays all fungicides are subject to exponential decay, with

$$
\frac{d C}{d t}=-\delta C
$$

in which the decay rate $\delta$ depends on the fungicide in question.
Epidemiological effects of fungicide and dose-response. The effect of a fungicide on a pathogen depends upon its mode of action, which differs between chemicals. Protectant fungicides are assumed to affect the pathogen's rate of infection, whereas eradicant fungicides affect the rate at which latently infected tissue becomes infectious (Hobbelen et al. 2011b). Fungicides that act as a combined protectant and eradicant affect both rates.

We use the symbol $\varepsilon$ to represent the proportionate effect of a given concentration of fungicide $(C)$ on a relevant rate parameter in our model (i.e., the rate of infection or the rate of emergence of infectivity). We assume the size of the effect depends on the chemical concentration via an exponential dose-response curve (Hobbelen et al. 2011b), and so take the functional form of the proportionate effect $\varepsilon(C)$ to be

$$
\varepsilon(C)=\omega\left(1-e^{-\theta C}\right)
$$

The parameters $\omega$ (maximum effect) and $\theta$ (curvature) vary between fungicides. For mixtures, we assume independent action

$$
1-\varepsilon\left(C_{1}, C_{2}\right)=\left(1-\varepsilon\left(C_{1}\right)\right)\left(1-\varepsilon\left(C_{2}\right)\right)
$$

in which $C_{1}$ and $C_{2}$ are the individual concentrations (Hobbelen et al. 2011a).

Modeling Septoria leaf blotch on winter wheat. Description of the model. We adapt a previously validated, compartmental model of Septoria leaf blotch (Z. tritici) on winter wheat over successive growing seasons (Fig. 1). The model is semidiscrete, tracking pathogen dynamics in continuous time within each growing season, linking seasons via sharp discontinuities at season boundaries (Hilker et al. 2017; Mailleret and Lemesle 2009). Parameterization of the model is fully described in Hobbelen et al. (2011a,b; 2013), in which the procedure for testing the fitted model against field data are also reported. The set of equations defining the model is given in the Appendix; we concentrate here on summarizing its important features.

The model tracks the leaf area index (LAI), the area of leaf per unit area of ground, for the upper five leaves of wheat plants (van den Berg et al. 2016). The model distinguishes healthy, uninfected leaf tissue (Susceptible) from different classes of infectious tissue: latently-infected (Exposed), sporulating (Infectious), and dead (Removed). The dynamics of resistance is tracked by separating fungicide-sensitive and fungicide-resistant leaf tissue (for example, splitting the infectious compartment into $I_{R}$ and $I_{S}$ ).

There are large variations in the LAI presented by a wheat crop over a single season, and this affects epidemiological dynamics (Cunniffe et al. 2015). The model accounts for this by including time-dependent rates of production of healthy tissue and of natural leaf senescence, allowing faithful representation of the top five leaves. The model also accounts for decaying inoculum on lower leaves (Primary), which initiates seasonal epidemics on the upper leaves. The amount of tissue in each compartment is reset to its initial value at the beginning of each season, with the ratio of fungicide-resistant to fungicide-sensitive inoculum set according to the corresponding ratio in the infectious compartments at the end of the previous year (or to the assumed initial frequency of resistance in the first year).

Fungicide effects and timing. We follow Hobbelen et al. (2011a) in taking pyraclostrobin as the high-risk fungicide and chlorothalonil as the low-risk fungicide; this risk designation for the two chemicals is supported by the current guidelines of the Fungicide Resistance Action Committee (FRAC) (Brent and Hollomon 2007). We assume that pyraclostrobin acts as a combined protectant and eradicant, but that chlorothalonil has only protectant activity. We consider two applications of fungicide per season, with a T1 spray at Zadoks growth stage 32 (second node formed in main stem, corresponding to emergence of leaf three) and a T2 spray at growth 
stage 39 (emergence of the flag leaf). These timings are representative of those used in winter wheat growing areas (Paveley et al. 2014).

Calculating yield. We estimate the relative yield $(Y)$ by integrating the amount of photosynthetically-active leaf area (i.e., tissue which is either uninfected or which has not yet started to support sporulation, so is in class $S$ or $E$ in our model) over a critical period for grain formation, which here we take to be from growth stage 61 to growth stage 87 on the Zadoks scale (Gooding and Dimmock 2000; Waggoner and Berger 1987)

$$
Y=\frac{\int_{T_{G S 61}}^{T_{G S 7}}\left(S(t)+E_{R}(t)+E_{S}(t)\right) d t}{\int_{T_{G S 61}}^{T_{G S 87}} S_{\text {disease-free }}(t) d t} \times 100 \%
$$

The denominator normalizes the yield to that obtained from a disease-free crop (in which case the two $E$ classes can be omitted from the integrand, since there is no disease in the system).

Tactic performance. The goal of any anti-resistance tactic is maintaining effective disease control. However, this begs a question: what level of control is effective? We define a threshold level of disease beyond which management is considered to have failed, taking a 5\% yield loss as the critical level growers will tolerate (Hobbelen et al. 2011a). The effective lifetime of the highrisk fungicide (the "usefulness time" of van den Bosch and Gilligan
[2008]) is defined as the number of seasons until this critical yield loss occurs. We use the following metrics.

- Selection ratio (SR). Proportional increase in the frequency of resistance over the first season, i.e., the proportion of the total infectious tissue $\left(I_{R}+I_{S}\right)$ infected by the resistant strain $\left(I_{R}\right)$. This measures the rate at which fungicide resistance spreads initially.

- Lifetime yield (LY). Total within-season yield over the entire effective lifetime. This allows tactics that have similar effective lives but differences in within-season performance to be distinguished.

Comparison of tactics depends on the metric. For selection, we define $Z=\mathrm{SR}_{\mathrm{ALT}} /\left(\mathrm{SR}_{\mathrm{ALT}}+\mathrm{SR}_{\mathrm{MIX}}\right)$, in which $\mathrm{SR}_{\mathrm{ALT}}$ is the selection ratio of the best-performing of the two alternation tactics, and $\mathrm{SR}_{\mathrm{MIX}}$ is that of mixtures. Since smaller values of the selection ratio are superior, values of $Z$ lower than 0.5 indicate alternation is preferred. For lifetime yield, larger values indicate a better performing tactic, and we preserve the directionality of the $Z$ metric by instead defining $Z=\mathrm{LY}_{\mathrm{MIX}} /\left(\mathrm{LY}_{\mathrm{ALT}}+\mathrm{LY}_{\mathrm{MIX}}\right)$, again using the bestperforming alternation tactic in the comparison.

Effect of model structure. We check the robustness of our results to the set of mechanisms included in the underlying epidemic model. We identify three components that could be significant.

- Host-limited infection. The density of host tissue is modeled in some detail, with a complex time-dependent function
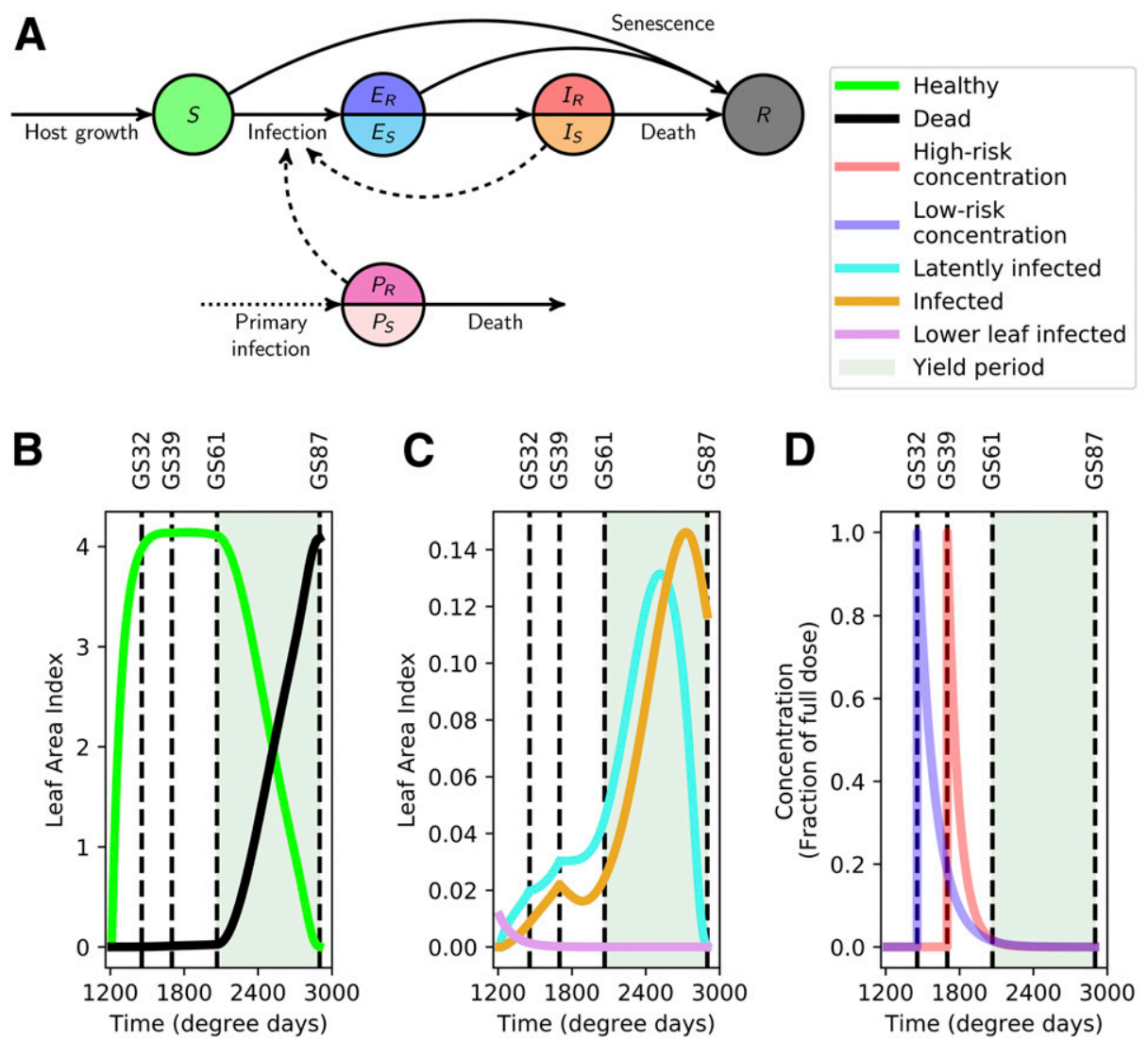

Fig. 1. Model of Septoria leaf blotch on winter wheat. A, A schematic showing the structure of the within-season model for Septoria on winter wheat. The model distinguishes healthy, uninfected leaf tissue (Susceptible) from different classes of infectious tissue: latently-infected (Exposed), sporulating (Infectious), and dead (Removed), as well as tracking the density of inoculum on lower leaves (Primary). Circles represent these epidemiological compartments (split into two where necessary to account for fungicide-sensitive and fungicide-resistant pathogen strains), solid lines represent transitions between compartments, dashed lines represent effects on the rates of transitions, and the dotted arrow represents the point of initial infection in each growing season. Panels B, C, and D show the dynamics of the model in the first growing season using the default model parametrization and the alternation low-high tactic at full doses of both chemicals. This corresponds to a full dose of the low-risk fungicide at GS32 (1,456 degree days after planting), and a full dose of the high-risk fungicide at GS39 (1,700 degree days after planting). The critical time for the accumulation of yield (GS61 to GS87; 2,066 to 2,900 degree days after planting) is shaded; control is considered to have broken down if yields $<95 \%$ of the disease-free yield are obtained. B, The leaf area index of healthy and dead tissue over time. C, The amount of primary inoculum and leaf area index of infected tissue over time. D, The concentration of both fungicides over time. Note that in panels B, C, and D, dynamics start 1,212 degree days after the start of the growing season; this corresponds to the time of emergence of leaf five, with all pathogen dynamics before that time subsumed into the initial condition for the primary inoculum. 
representing production of susceptible host tissue, whereas simpler models use exponential growth.

- Latent period. New infections only become infectious after a latent period, whereas in simpler models infected tissue becomes infectious immediately.

- Phenology. The model includes a complex treatment of withinseason timing, with primary inoculum from lower leaves initiating upper leaf epidemics, and also the senescence of living leaf tissue. These features are absent from the simpler models.

These complexities are sequentially taken out of the model and the analyses rerun (the procedure for this is described in full in the Appendix).

Effect of pathosystem. Modeling powdery mildew on grape. As a further test of robustness, we repeat a selection of analyses using a model of powdery mildew (E. necator) on grapevine (Burie et al.2011) (Fig. 2). In addition to being previously parameterized to match the grapevine powdery mildew pathosystem, there are three additional differences in model structure in comparison with the Septoria model.

1. There is no primary inoculum compartment, and epidemics are instead initiated by a small amount of tissue being set to be latently infected (i.e., exposed) at the start of each season.

2. An additional compartment is included in the model, accounting for leaves developing Ontogenic resistance by virtue of age.

3. The model includes shoot-topping, in which upper shoots are removed to encourage secondary shoot growth.
Full details of the model, and its parameterization, are given in the Appendix.

We selected the Burie et al. (2011) model as a previously published, parameterized and validated model of this pathosystem that could be used without change as a baseline to which the complexity of fungicide resistance management could be added. We could have extended the model to include a number of aspects that are potentially epidemiologically important in some regions, for example, an ongoing source of primary infection, or manual or mechanized leaf picking. However, this would then require refitting and revalidating the model, and given our focus here on the dynamics of fungicide resistance, we did not pursue any such changes.

Fungicide effects and timing. For powdery mildew we model trifloxystrobin as the high-risk fungicide, and sulfur as the low-risk fungicide, assuming both chemicals combine protectant and eradicant modes of action (Reuveni 2001). We assume flowering occurs at day 163 of the season (Mammeri et al. 2014), and that spraying is done either side of this, 2 days before and 12 days after flowering. This is fewer sprays than normally used in French viticulture (Calonnec et al. 2006; Savary et al. 2009), although it is within the range leading to acceptable control (Gadoury et al. 2003).

Effective lifetime and yield. The Burie et al. (2011) model tracks the severity of powdery mildew on grapevine leaves. However, prices obtained by a grower would depend on a combination of yield and grape quality for winemaking. Effects of leaf infection upon yield and quality are complex (Calonnec et al. 2004; Pool et al.
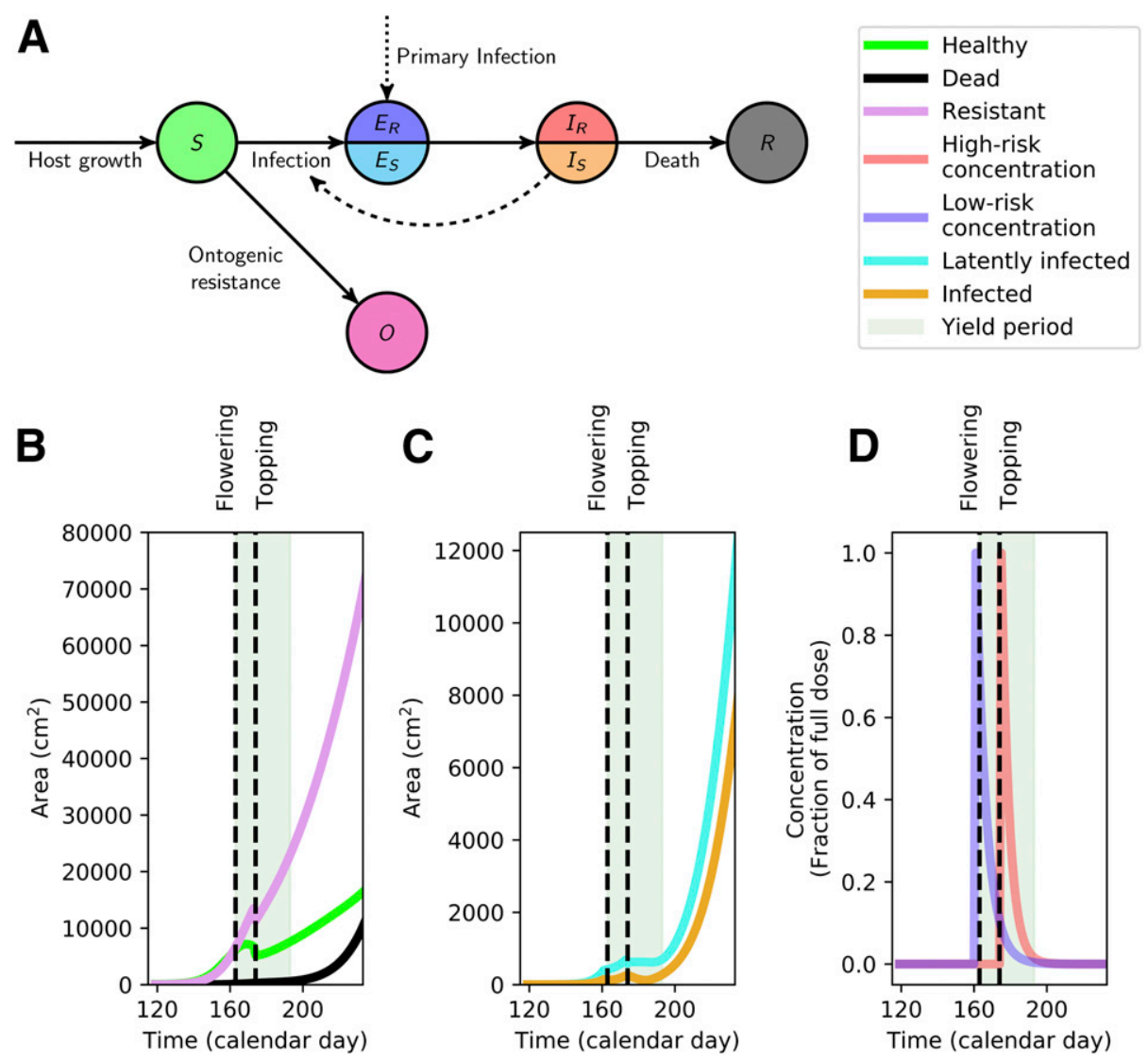

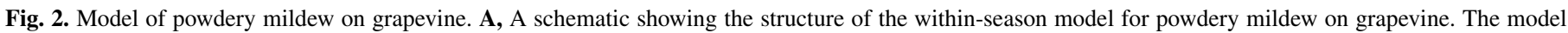

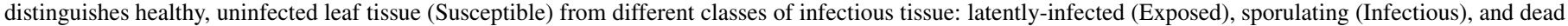

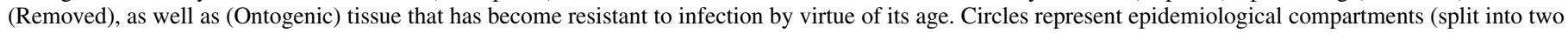

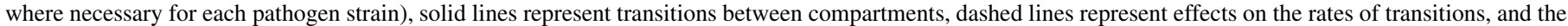

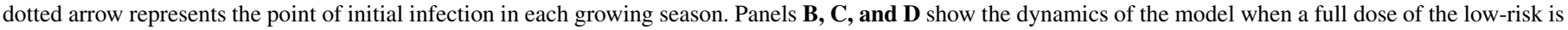

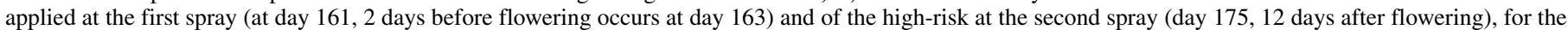

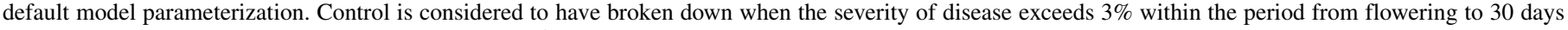

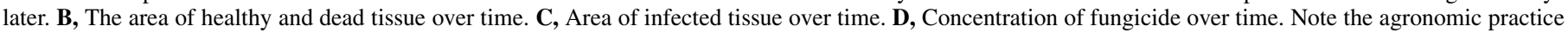

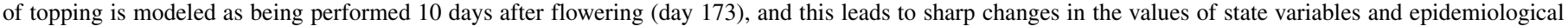
parameters at this time. 
1984). Quantifying fine details of this would require a more detailed treatment than would be appropriate here. However, there is a strong positive correlation between leaf infection and berry infection (Calonnec et al. 2006; Delière et al. 2015). We therefore simply use the level of leaf infection as a proxy for yield, taking $3 \%$ as the critical threshold on the peak level of berry infection within 30 days of flowering beyond which control is considered to have broken down, since berries are almost entirely resistant after this period (Gadoury et al. 2003). Similarly, stringent thresholds are common in French viticulture (Deliere et al. 2010). We then set the equivalent of lifetime yield to be the effective lifetime of the high-risk fungicide, i.e., the number of seasons until this critical threshold is exceeded.

\section{RESULTS}

Initial disease control at full doses. For Septoria leaf blotch and applying full doses of both fungicides, all three tactics lead to adequate control in the first season (yield $>95 \%$ of the disease-free yield) (Fig. 3A). Optimal initial control is obtained under mixture ( $\sim 97.4 \%$ yield), with lower yields from both alternation tactics ( $\sim 96.0$ and $96.4 \%$ for low-high and high-low, respectively). The first-season yield is highest for mixture because of the concave dose-response curve, with diminishing returns from increased concentrations. Spraying half the dose twice as often therefore leads to better control (recall full dose corresponds to half dose at both sprays under mixture). The alternation high-low tactic slightly outperforms the low-high tactic in the first season since the high-risk fungicide is assumed more efficacious (maximum effect $\omega_{H}=1.0>0.48=\omega_{L}$ ). All other things being equal, control is improved by applying the high-risk fungicide earlier, since it then targets the pathogen when its relative growth rate is larger.

Evolution of resistance at full doses. For all three tactics, there is a sharp breakdown of control after $\sim 15$ seasons (Fig. 3A). This is driven by a rapid increase in the proportion of the resistant strain, which increases sigmoidally from being practically undetectable $(<1 \%)$ to near fixation $(>99 \%)$ within one or two growing seasons (Kable and Jeffery 1980) (Fig. 3B). Disease control then rests entirely on the low-risk fungicide, and all three tactics become ineffective (i.e., yield $<95 \%$ ). Due to dose-splitting and the concave dose-response curve, when resistance is at high frequency the best yield is then obtained under mixture (Fig. 3A), although this level of control is not economically viable. The improved performance of the low-high tactic relative to high-low alternation after resistance has taken over is again due to timing: control is improved by applying the sole effective fungicide earlier in each season.
Although the timing of the sharp increase in the frequency of fungicide-resistant pathogen is similar for all three tactics, it occurs earliest for mixture then for alternation high-low then for low-high (Fig. 3A and B). This is precisely the order of the efficacy of the tactics for disease control in the first season. Applying fungicides as a mixture therefore leads to slightly more effective disease control, but in part as a consequence of this, exerts a stronger selective pressure. Considered over the effective lifetime, the alternation lowhigh tactic therefore has the highest lifetime yield (Fig. 3C). At full dose, however, differences between the tactics are relatively minor.

Responses of selection and lifetime yield to dose. Full dose results illustrate how disease control and selection are closely related. We therefore consider performance over a range of dose combinations, identifying how to select a pair of doses, as well as a spray tactic, to optimally balance control against selection. For selection, there are dose combinations favoring both alternation and mixture (Fig. 4A). Alternation exerts less selection than mixture at higher doses of high-risk $\left(C_{H}\right)$. The concave dose-response means that at high doses the effect on pathogen growth rates of the half dose under mixture approaches that of full dose under alternation. However, under mixture this dose is applied twice as often, and selection occurs for longer. Conversely, at higher doses of the lowrisk fungicide $\left(C_{L}\right)$, mixture tends to be preferred, since alternation receives no suppression of pathogen growth rate from the low-risk at the time the high-risk fungicide is applied.

Patterns in lifetime yield are more complex (Fig. 4B). There is a region of dose-space (hatch-shaded gray) within which no tactic leads to sufficient control even before resistance has spread. This outcome is associated with low doses of both chemicals, although even at full dose of low-risk, some high-risk is required (recall the high-risk chemical is the more efficacious). There is an intermediate region (shaded dark green) within which, at the same doses of both chemicals for each tactic, effective control is only possible under mixture because of dose-splitting. For larger doses of both chemicals either mixture (shaded light green) or one of the alternation tactics (shaded light brown) can have the best performance, or there can be approximately equal lifetime yields (within 1\%; shaded white).

Selecting an optimum tactic and dose. The largest lifetime yield over all tactics and pairs of doses is marked with the red arrow on Figure 4B. It corresponds to spraying a mixture of a full dose of low-risk with a dose of high-risk slightly larger than that required for economically acceptable yield in the first season. As the low-risk fungicide exerts no selection, it is unsurprising that the maximal permissible amount of low-risk should be optimal, since this allows the smallest amount of high-risk to be applied while maintaining acceptable disease control. However, it is less obvious why the optimal tactic should be to apply the high-risk fungicide as part of a mixture, and why this particular dose of high-risk
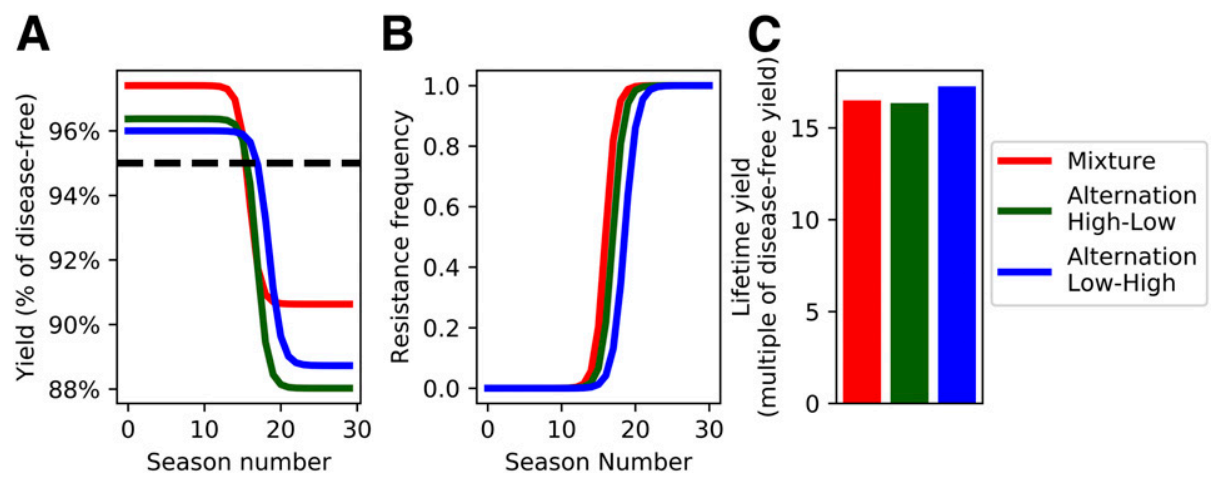

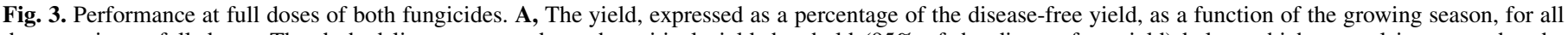

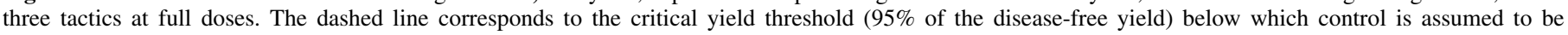

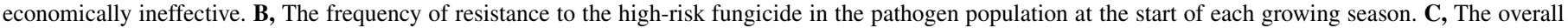
lifetime yield (expressed as a multiple of the disease-free yield in a single season). 
(i.e., just above the amount required to ensure effective control) is required.

We therefore examine responses to the dose of high-risk $\left(C_{H}\right)$ with the low-risk fixed at full dose under all three spray tactics (Fig. $4 \mathrm{C}$ to $\mathrm{E}$ ). This corresponds to the vertical line in dose-space $C_{L}=1.0$ in Figure 4A and B. As already noted, for a given value of $C_{H}$, mixture leads to the best initial disease control because of the beneficial effect of dose-splitting (Fig. 4C). The implication is that lower $C_{H}$ can maintain adequate control under mixture $\left(C_{H} \geq 0.29\right.$; dashed line in Figure 4C) compared with either alternation $\left(C_{H} \geq\right.$ 0.44 or 0.56 ). The response of the strength of selection to $C_{H}$ is more complex, with both mixture and alternation potentially leading to smaller selection ratios at different $C_{H}$ (Fig. 4D).

To understand optimum performance in more detail, we compare the selection ratios at the low end of permissible doses for each tactic, since these maximize lifetime yields (Fig. 4E). For the model and parameterization used here, the lower permissible dose under mixture outweighs the effect of spraying the high-risk fungicide twice as often, and exerts less selection than the lowest permissible doses under either alternation (at 95\% yield in the first season, $\mathrm{SR}=$ 2.28 for mixture versus 3.08 and 3.15 for alternation high-low and low-high, respectively). The lower selection ratio leads to a longer effective lifetime, and spraying the fungicides as a mixture optimizes lifetime yield.

The optimal dose of the high-risk fungicide $\left(C_{H}\right)$ is slightly higher than the minimum $C_{H}$ ensuring acceptable control in the first season. This is because the effective lifetime is discrete, leading to ranges of $C_{H}$ which all break down within the same season. Within any range of doses with the same effective lifetime, the optimum lifetime yield is often obtained by selecting a higher $C_{H}$, benefitting from slightly improved control in each season it remains effective. Too high a $C_{H}$ however can lead to more dramatic failure in the final season, and thus the optimal $C_{H}$ may not be the highest dose with the longest effective life. This is difficult to see in Figure 4E, but the "horizontal" parts of the response are not quite horizontal. The red arrow in Figure 4B is therefore above the boundary between the gray and dark green regions.

Balancing selection and control. We further dissect the trade-off between selection and control by considering equal doses of high- and low-risk (Fig. 5A to E), corresponding to a different visualization of results underpinning the line $C_{H}=C_{L}$ in Figure 4A and/or B. For all three tactics, as the dose of high-risk and low-risk is increased, both the first season yield (Fig. 5A) and selection ratio (Fig. 5B) increase. However, dose-splitting means that viable control is again retained under mixture at much lower doses. The overall maximum lifetime yield is therefore under mixture ( $\mathrm{LY}=$ 19.1, $C_{L}=C_{H}=0.5$ ) (Fig. 5C). Tactic performances can be normalized against each another by replotting the selection ratio and lifetime yield as a function of the first season yield (Fig. 5D and E). This reiterates that, at least at the same level of initial disease control and using equal proportions of both chemicals, mixture leads to less selection (Fig. 5D) and a larger lifetime yield (Fig. 5E). Differences between tactics are small, however.

Examining responses of selection and control to first season yield is a convenient mechanism to allow results at all doses, not just when $C_{H}=C_{L}$, to be visualized (Figs. $5 \mathrm{~F}$ to $\mathrm{H}$ ). For any given initial level of control, mixture can produce higher lifetime yields and lower selection ratios (Fig. 5F and $\mathrm{G}$ ). However, mixing fungicides can also produce lower yields and higher selection ratios. The variation in selection and yield for a given level of control is therefore much larger with mixture than with either alternation tactic. Examining tactics that have the same initial disease control shows that mixtures can produce any particular effective lifetime at much lower $C_{H}$. This is shown in Figure $5 \mathrm{H}$, which-for the ranges of doses of both fungicides under all three tactics which lead to first season yields between 95.45 and $95.55 \%$ - shows values of $C_{H}$
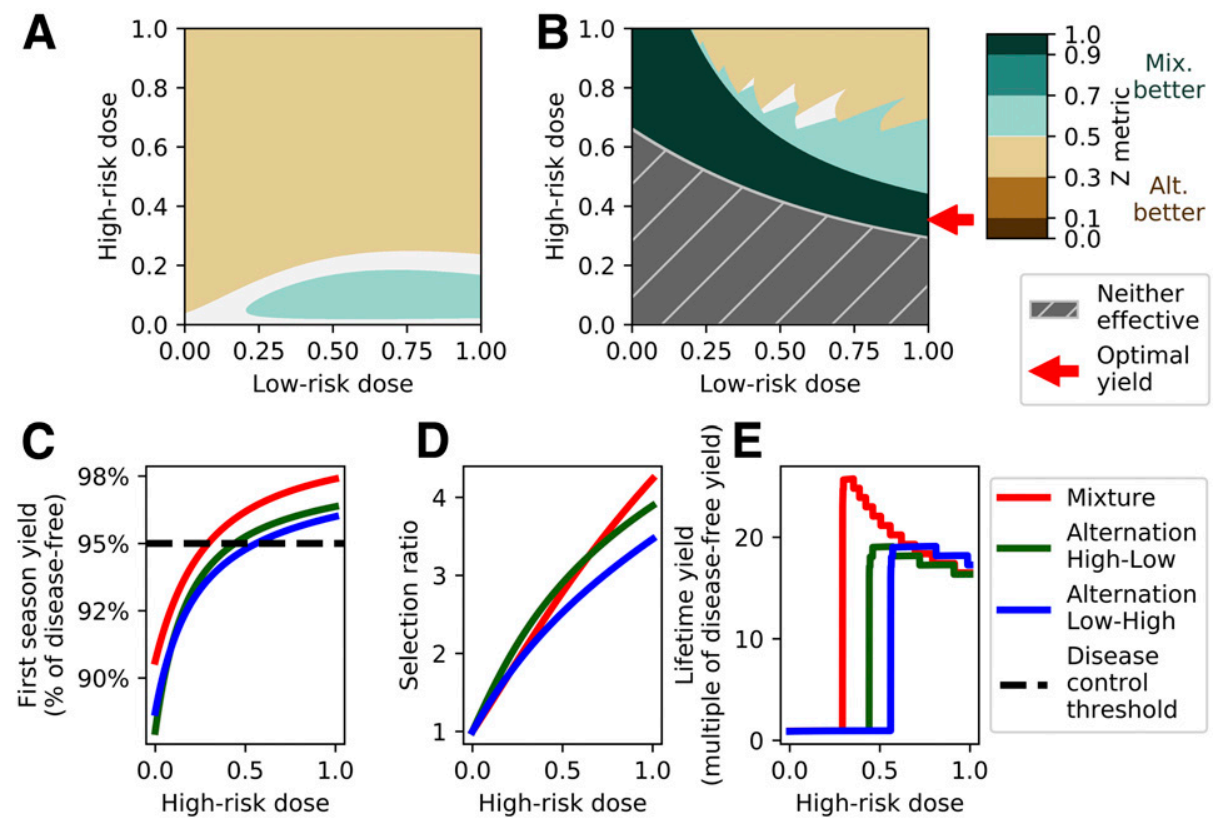

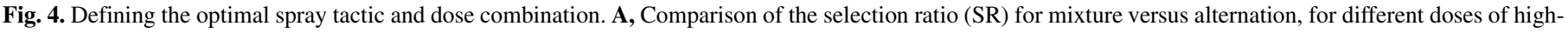

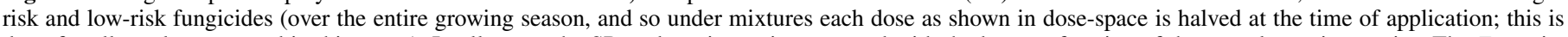

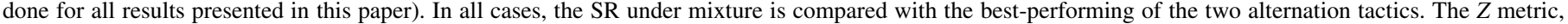

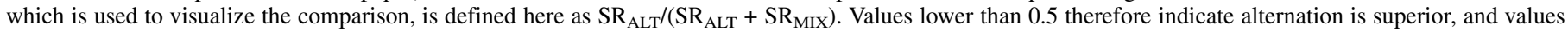

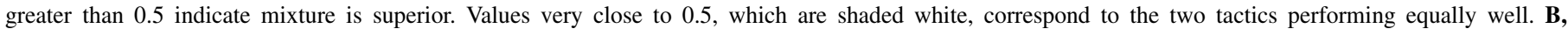

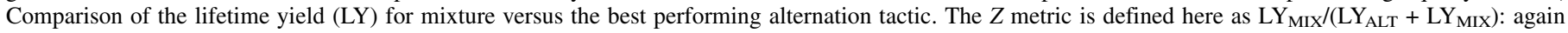

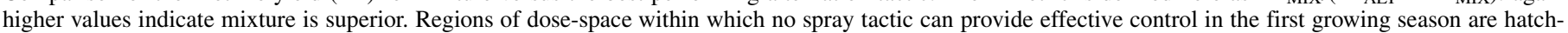

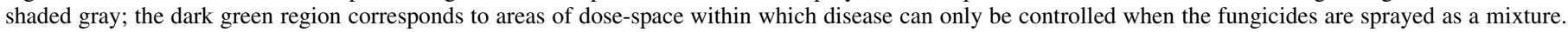

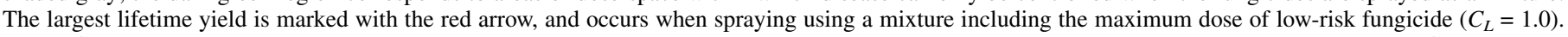

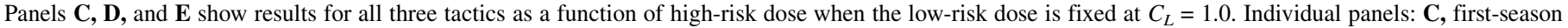
yield; D, selection ratio; and $\mathbf{E}$, lifetime yield. In $\mathbf{C}$ the dashed line shows the disease control threshold. 
leading to each effective lifetime (i.e., a vertical slice through the data underpinning Figure 5G).

Effect of epidemiological and fungicide parameters. Results thus far correspond to a single model parameterization. We test robustness by altering values of a number of key parameters. In all cases the dose of low-risk fungicide is fixed to be maximal (i.e., $C_{L}=1.0$ ), as we have identified no mechanism by which changing parameter values can cause this not to be optimal. We then consider the response of lifetime yield to changing $C_{H}$.

As an example, we examine in some detail the effect of the infection rate ( $\beta$ ) (Fig. 6A). If $\beta$ is made significantly larger than the default, all three tactics fail to give sufficient control at any $C_{H}$ (dark gray hatching). If $\beta$ is made sufficiently smaller, then control can be maintained indefinitely through the low-risk fungicide alone (light- gray hatching). Both cases are unrealistic. Within the realistic range of values of $\beta$, exactly the same pattern is seen as before. At low $C_{H}$, no spray tactic can provide effective control. At slightly higher $C_{H}$ mixture performs best. At the highest $C_{H}$ alternation performs better, although for large infection rates this might require $C_{H}>1.0$ (i.e., a dose above the permissible maximum label dose). As $\beta$ is increased, the threshold $C_{H}$ at which mixture first becomes effective shifts upwards, as more fungicide is required to provide acceptable disease control.

The optimal dose is always lower for mixture than for either of the alternations (Fig. 6B; the saw-tooth pattern is because the effective lifetime is discrete). The corresponding optimal lifetime yield is always larger under mixture (Fig. 6C), and for all tactics corresponds to selecting $C_{H}$ close to the threshold required for
A

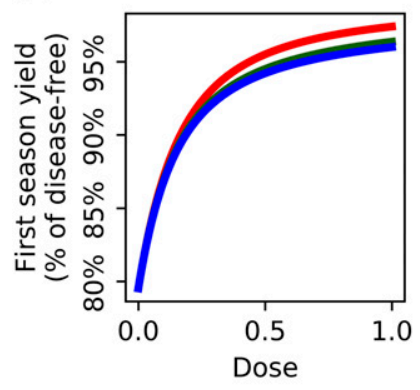

D

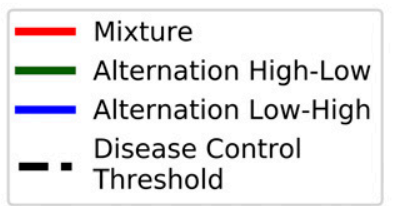

B

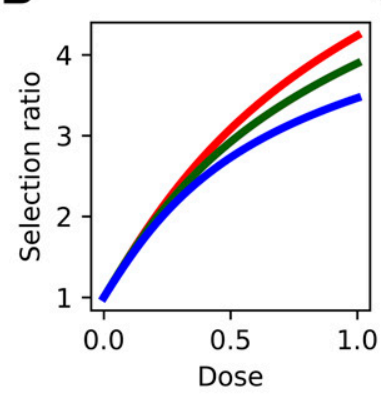

C 흠

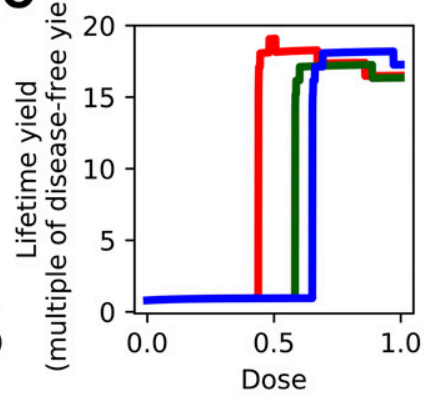

(D)

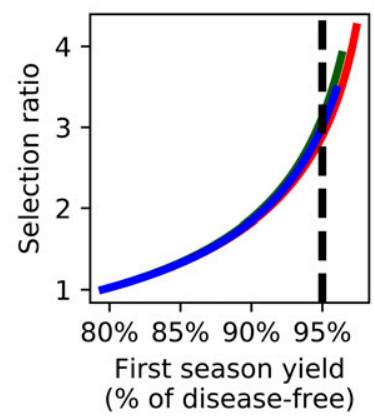

E 음

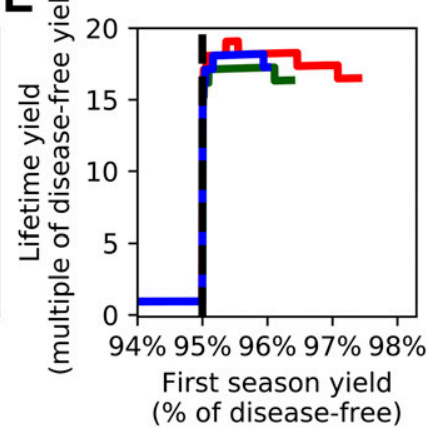

F

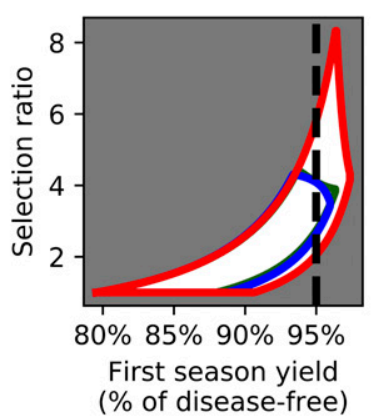

G

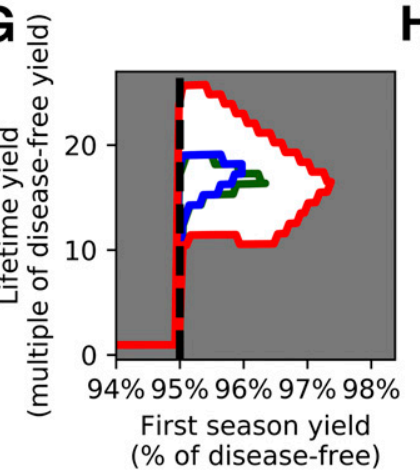

H

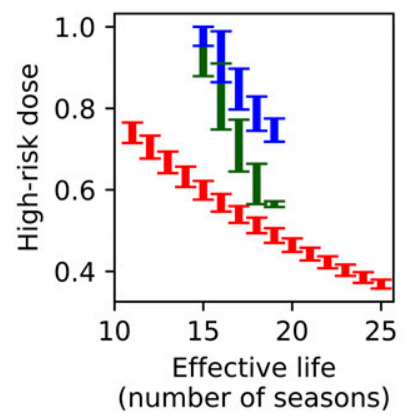

Fig. 5. Balancing selection and disease control. Panels $\mathbf{A}, \mathbf{B}$, and $\mathbf{C}$ show performance as the dose varies but when equal amounts of low- and high-risk fungicide are sprayed (i.e., $C_{L}=C_{H}$ ). Individual panels are: $\mathbf{A}$, first-season yield; $\mathbf{B}$, selection ratio; and $\mathbf{C}$, lifetime yield. Panels $\mathbf{D}$, (selection ratio) and $\mathbf{E}$, (lifetime yield) show the results from $\mathbf{B}$ and $\mathbf{C}$ plotted as a function of the first season yield. When normalized for the level of initial disease control, mixture leads to less selection and larger lifetime yields than either alternation tactic. Panels $\mathbf{F}, \mathbf{G}$, and $\mathbf{H}$ show the results when the constraint that doses of both fungicides should be equal is removed. For all three tactics there are ranges of values of the selection ratio and the lifetime yield that correspond to each single value of the first season yield, with different relative proportions of high-risk to low-risk fungicide that is sprayed. The top and bottom of the ranges of the selection ratio and the lifetime yield are shown for each tactic, although all intermediate points can be attained for different combinations of low- and high-risk chemicals. The ranges are wider for mixture than for either alternation tactic, meaning that, depending on the dose of high-risk fungicide, mixture can lead to both better and worse outcomes for resistance management at any level of disease control. However, dose combinations that cause mixture to provide the most effective resistance management can always be selected. H, The range of high-risk doses that lead to different effective lifetimes, for each tactic (first season yields between 95.45 and $95.55 \%$; this corresponds to vertical slice through the data shown in panel G). A wider range of effective lifetimes are possible at this level of disease control under mixture, and a given effective lifetime results from a smaller dose of high-risk fungicide. A, D, E, F, and G, Dashed line shows the disease control threshold. 
effective first season control (Fig. 6D). For all values of the infection rate, $\beta$, the optimal tactic is therefore again to spray a little more high-risk fungicide than required for effective control in the first season, and to do so using a mixture of fungicides on each spray.

The pattern is consistent for all parameters tested in our sensitivity analysis (Fig. 7). For parameters which cause disease to spread faster as they are increased, more high-risk fungicide is required for effective control, and the characteristic pattern shifts upward (Fig. 7C and F). Conversely, for parameters for which an increase leads to decreased rates of disease spread, a smaller amount of high-risk is permissible (Fig. 7A, B, D, E, and G). Changing the initial frequency of resistance has a negligible effect on the relative performance of the tactics in the first season, for both resistance management and yield. However, at higher initial levels of resistance, control fails sooner, increasing the importance of disease control in the earlier seasons for the lifetime yield and thus favoring mixture (Fig. 7H).

Effect of model structure. To facilitate intermodel comparison, we return to comparing tactics in dose-space. The simplest model, with both pathogen strains growing exponentially, is similar to models used in the early fungicide resistance modeling literature (Delp 1980; Kable and Jeffery 1980; Skylakakis 1981). Indeed, if we additionally assume fungicides do not decay, an analytical prediction of which tactic leads to better resistance management at a given pair of doses can be generated. The other models are too complicated for mathematical analysis, although the same pattern is seen for selection in dose-space in every model (Fig. 8). The only real differences between models are the slightly larger regions within which alternation provide better resistance management when models include a latent period. When a latent period is not included, the high-risk fungicide loses its eradicant mode of action, and so becomes generally less efficacious. As with dose and fungicide parameter values, less effect from the high-risk fungicide then favors mixture.
In the models that include host-limited infection, and thus the loss of host tissue to disease, we also investigate how predictions of lifetime yield are affected by model structure (Fig. 9). Predictions vary between models, which is perhaps unsurprising given the additional complexity underlying the yield metric. However, although the patterns vary, the characteristic pattern in dose-space is conserved. At low $C_{H}$, both tactics fail to give acceptable yield, at slightly higher doses mixture outperforms either of the alternation tactics, and at the highest doses alternation outperforms mixture. Exactly as before, the optimal tactic is therefore again to spray a little more high-risk fungicide than is required for effective control in the first season, and to do so under mixture.

Effect of pathosystem. Results for powdery mildew are similar to those for Septoria for both comparisons in dose-space, with alternation performing better at higher doses of the high-risk fungicide in terms of both resistance management and long-term yield (Fig. 10A and B). Mixture performs increasingly well for selection if $C_{L}$ is increased, while the yield metric produces more complicated patterns, again as before. Compared with the Septoria model, the boundary between the areas where mixture and alternation perform better for selection curves in the opposite direction; concave upward rather than downward (compare Figure 10a with Figure 4A). This is because the maximum effectiveness of the low-risk in the powdery mildew model is larger than its counterpart in the Septoria model (compare analytic predictions from the simple exponential growth model, given in Supplementary Text S1). Again, at full dose of both fungicides the superior tactic for both resistance management and long-term yield is alternation low-high.

However, when normalizing tactic performance by the level of initial control (Fig. 10C and D), mixture is again capable of generating lower selection pressures and higher effective lives. Compared with Septoria, the alternation tactics have less overlap in their performance and the worst-yielding mixture tactics are much more similar to the worst-yielding alternation. Nevertheless, the key
A
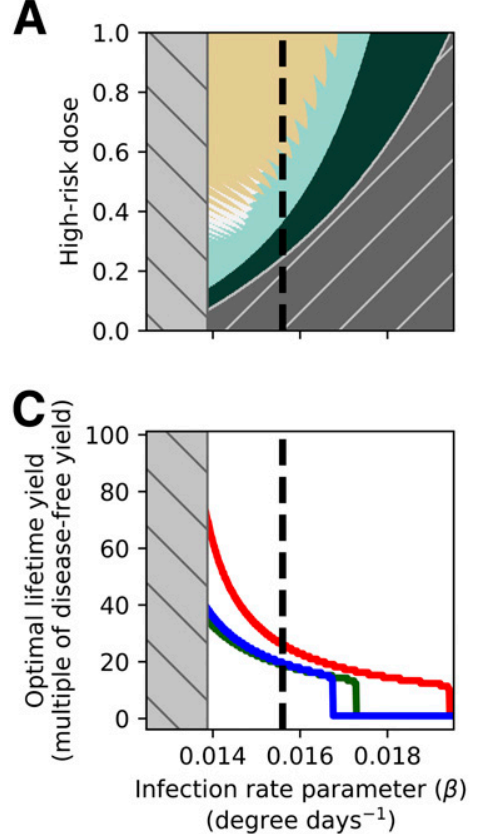

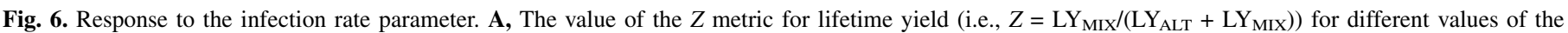

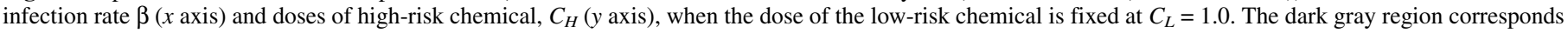

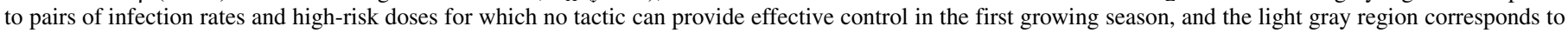

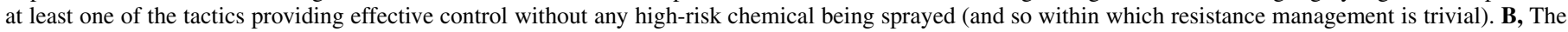

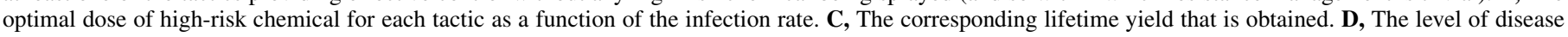

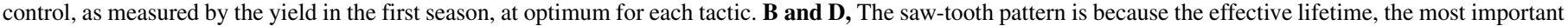
determinant of the lifetime yield, is a discrete quantity. The dashed lines in all panels shows the default value of $\beta$. 
result is that the overall optimal tactic for long-term yield is, yet again, to apply as much low-risk as possible, combined with slightly more high-risk than needed for an acceptable initial level of disease control, and to do so under mixture.

\section{DISCUSSION}

We considered resistance management of a fungicide at high-risk of resistance, comparing performance of combining the high-risk chemical with a low-risk fungicide sprayed as either a mixture or in alternation. We assessed performance via the lifetime yield before control breaks down due to fungicide resistance, performing four distinct comparisons. The simplest comparison considered full label doses of both chemicals. For Septoria, the largest lifetime yield was obtained by spraying in alternation, although the improvement relative to mixture was relatively small (Figs. 3 and 4A and B). Alternation was also optimal at full doses in our model of grapevine powdery mildew (Fig. 10A and B). While performance at full doses of both chemicals is a simple comparison, it is now common practice in some countries for fungicides to be used routinely at lower doses (Jørgensen et al. 2017). Additionally, the comparison depends strongly on model parameterization. By altering values of epidemiological and/or fungicide-performance parameters, either mixture or alternation can optimize lifetime yield at full dose ( $c f$. changes in color along the top of individual panels in Figure 7). The set of mechanisms included in the underlying epidemiological model can also affect whether alternation or mixture is the best tactic (note how closely regions shaded light green approach the top right points at which $C_{H}=C_{L}=1.0$ in some panels of Figure 9). Results of the full dose comparison are therefore equivocal, being system-, model-, and parameter-specific. There is also no guarantee that larger lifetime yields would not be obtained by spraying smaller amounts of fungicide, due to the smaller amount of selection that would thus be exerted.

Our second comparison therefore considered performance across all pairs of permissible doses. There are regions of dose-space within which either alternation or mixture optimize lifetime yield (Fig. 4B). The broad pattern in dose-space is robust to model structure (Figs. 8 and 9) and pathosystem (Fig. 10A and B). An underlying driver of the variation in performance at different doses is that, when normalizing by the applied doses, different tactics lead to varying levels of disease control in the absence of resistance (Figs. 4C and 5A). An alternate normalization, as in our third comparison, accounts for this by selecting combinations of pairs of
A

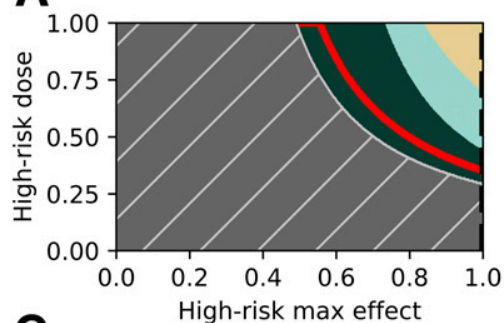

$$
\text { C }
$$

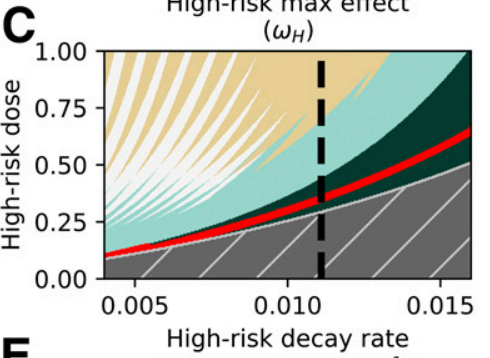

E
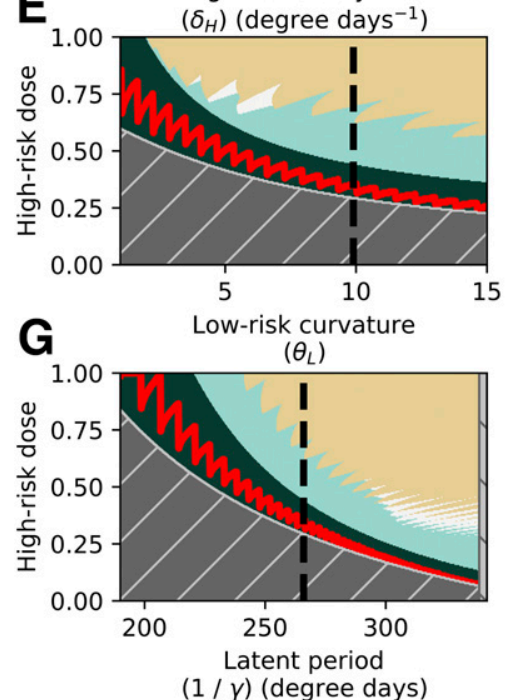

B

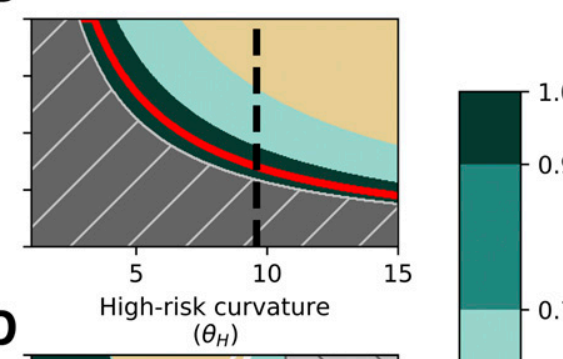

D

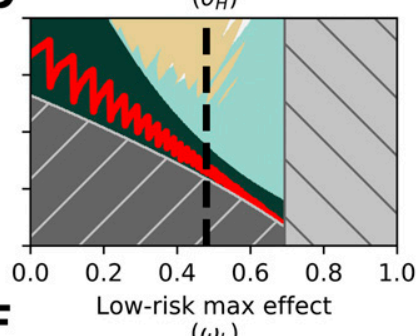

$\mathbf{F}$ $\left(\omega_{L}\right)$

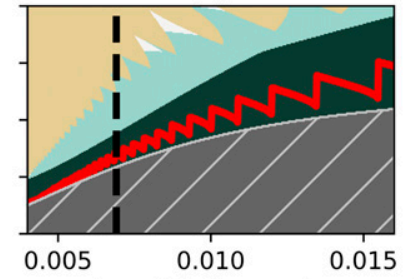

$\mathrm{H}$

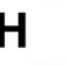

Low-risk decay rate

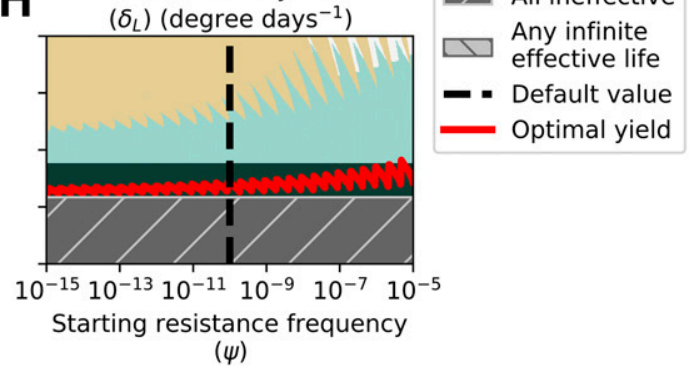

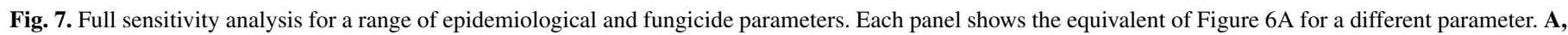

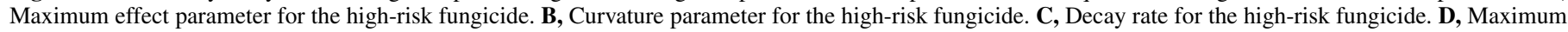

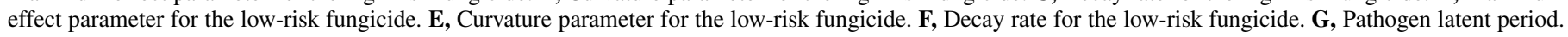

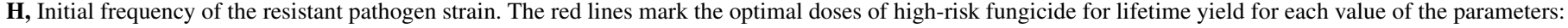
in all cases this corresponds to applying the two fungicides as a mixture. 
fungicide doses for each tactic that lead to identical initial levels of control (Fig. 5F and G). The effective lifetime and so lifetime yield for any given level of disease control then depends strongly on the amount of high-risk fungicide that is sprayed (Fig. 5H). Since the low-risk chemical exerts no selection in our model, it is optimal to include as much low-risk fungicide as possible in any spray program, and to combine this with as little high-risk fungicide as provides effective control (Figs. 4B and 10B). Arguably this is unsurprising (Shaw 2006), but focuses our attention on identifying the tactic which has the longest effective lifetime, and so the largest lifetime yield.

For this fourth and final comparison, for both pathosystems we considered (Figs. 4B and 10B), and for all model parameterizations
(Figs. 6 and 7) and sets of epidemiological mechanisms (Fig. 9) we tested, the maximum effective lifetime was obtained when fungicides were sprayed as a mixture. We found it was then always optimal to apply a full dose of low-risk mixed with close to the minimal dose of high-risk that retains effective control when there is no resistant pathogen. This optimizes the lifetime yield (red arrows in Figs. 4B and 10B, and red lines in Fig. 7). This spray program represents the true optimum of all tactics we considered.

Mathematical models of whether alternation or mixture is better for fungicide-resistance management have been developed for decades. In the early literature whether mixture provided any benefit beyond allowing a reduction in dose was contentious, and without explicit consideration of dose-response curves, the cost of

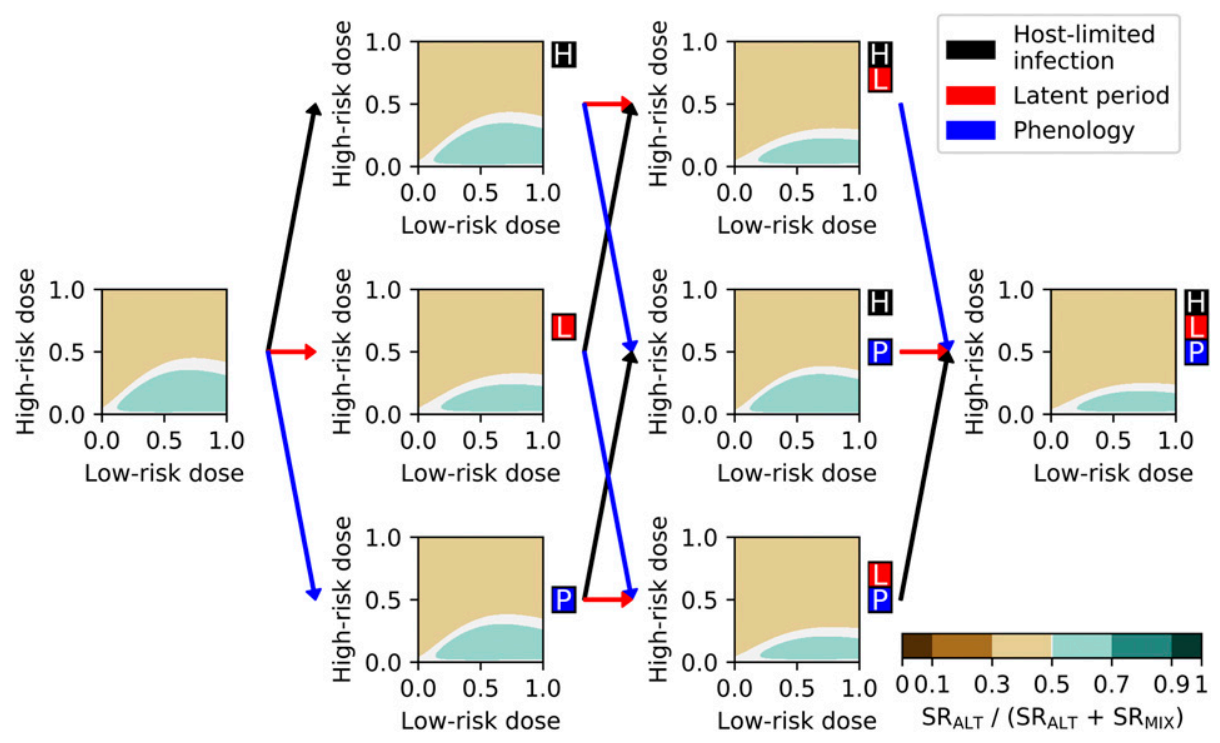

Fig. 8. Effect of model structure on the comparison between mixture and alternation for selection. The relative performance of mixture and (the best-performing) alternation is plotted in dose-space, by calculating the $Z$ metric, $Z=\mathrm{SR}_{\mathrm{ALT}} /\left(\mathrm{SR}_{\mathrm{ALT}}+\mathrm{SR} \mathrm{MIX}\right)$, for all of the Septoria submodels investigated. The colored boxes next to each subplot identify what model features are present, and the colored lines show which features differ between connected submodels. The right-most model includes all features, and so corresponds to the full model of Septoria considered in the bulk of the paper (i.e., the right-most plot is exactly as Figure 4A).

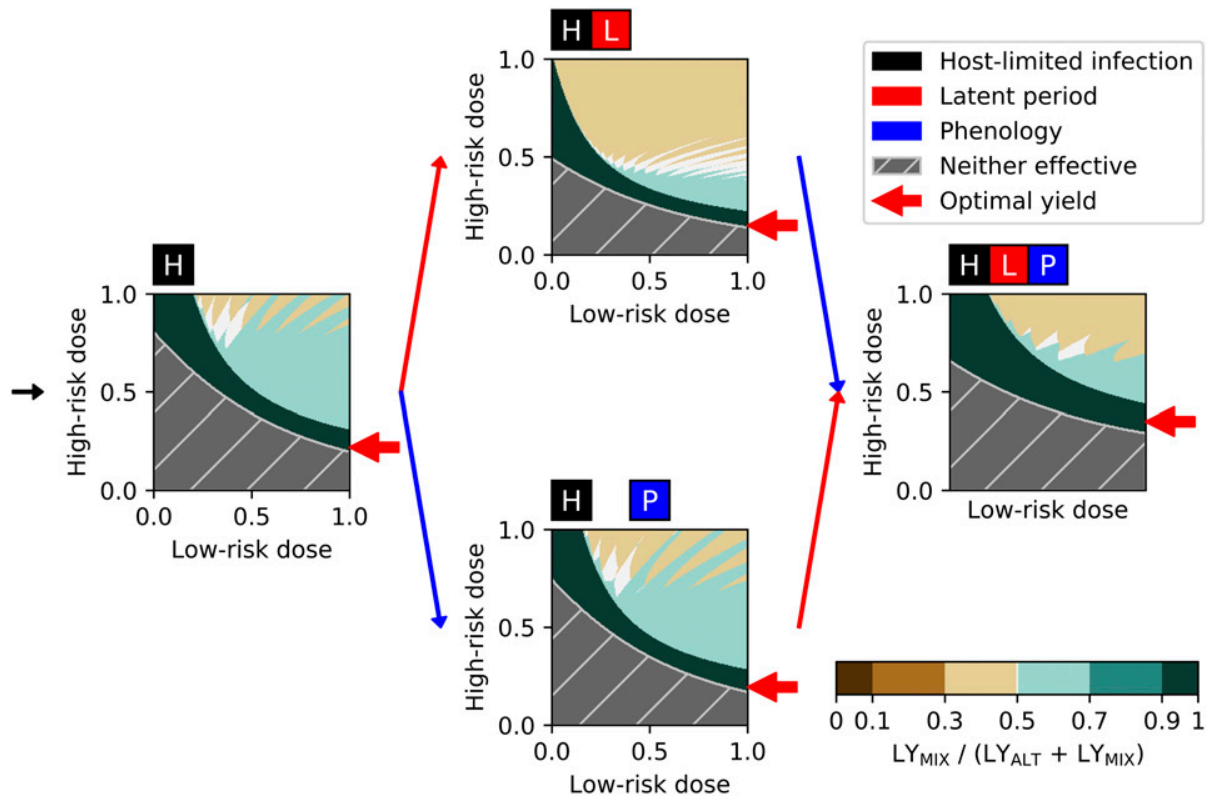

Fig. 9. Effect of model structure on the comparison between mixture and alternation for lifetime yield. The relative performance for lifetime yield of mixture and (the best-performing) alternation is plotted in dose-space, showing $Z=\mathrm{LY}_{\mathrm{MIX}} /\left(\mathrm{LY}_{\mathrm{ALT}}+\mathrm{LY}_{\mathrm{MIX}}\right)$, for a range of the Septoria submodels investigated. Note that it does not make sense to consider the yield in models that do not contain host limitation, and so these models are omitted. The optimal lifetime yield is marked by the red arrow (in all cases this is at full-dose of low-risk and is obtained under mixture). Note again that the right-most model corresponds to the full model, and so replicates Figure 4B. 
dose-splitting was not obvious. The recent formalization of the governing principles has allowed us to clearly disentangle the mechanisms driving the effect of fungicide mixtures on selection. The other major distinguishing feature of our work is our extensive sensitivity analysis to model parameterization as well as the pathosystem that is modeled. We also tested-via our structural sensitivity analysis to model structure-how the set of epidemiological mechanisms included in the underlying model affected our conclusions. We are not aware of other studies in the phytopathology disease modeling literature performing a similar comparison. Certainly, it is remarkable that the optimum over all tactics was independent of all these factors.

We have also shown how the governing principles originally introduced by Milgroom and Fry (1988) and more recently formalized by van den Bosch et al. (2014a) can be used to explain the behavior of resistance development in response to mixture and alternation. The driving mechanism underpinning the relative success of fungicide mixture is that dose-splitting of the low-risk fungicide gives better background disease control and permits a lower dose of high-risk to be used. The growth rate of the pathogen when the high-risk is applied is also suppressed by the low-risk, reducing selection further. For the set of models and parameterizations tested here these effects outweigh the negative effect of spraying the high-risk chemical twice as often. While we have not, and in general cannot, prove this will happen in all parameterizations of all models of all structures for all pathosystems, taken collectively our results provide extremely good evidence that applying fungicides as a mixture will be the best resistance management tactic in a range of situations. Furthermore, for a set of 1,000 parameter sets in which each parameter was sampled uniformly at random from the ranges shown in Figure 7, no case was found where mixture did not provide an overall better lifetime yield than alternation (the full procedure for this additional sensitivity analysis is described in the Appendix). We therefore feel justified in inferring on the basis of the evidence presented here that mixture will always be the best resistance management tactic for combining a low-risk and high-risk fungicide (with full resistance) when the objective is phrased in terms of optimizing the lifetime yield of the high-risk chemical, given that a threshold level of disease control must be attained in all years the chemical is sprayed.

The majority of our results are explained by dose-splitting and suppression by the mixing partner, both of which are simply explained by the governing principles. However, the fact that the two alternation tactics do not perform identically - and that these two tactics differ only in the order in which high-risk and low-risk chemicals are applied-shows that timing of fungicide application can also be important. These can likely also be explained by the governing principles, but with greater difficulty due to the nontrivial interactions between the end of the season, the growth rate of the pathogen at any given time, and the critical period for yield formation. We have therefore not pursued these differences here.

An aspect of resistance that we have not touched upon in our work is that resistance is often partial rather than full. This is highlighted by the fact that resistance is generally measured in terms of a resistance factor, the ratio of the $\mathrm{EC}_{50}$ values of the fungicide on resistant and sensitive strains. The resistance factor would be infinite if resistance were full. The models presented here could be readily extended to consider partial resistance by allowing the highrisk fungicide to affect the resistant strain, but with a reduced maximum effect or curvature parameter of the dose-response curve (Hobbelen et al. 2013; Mikaberidze et al. 2017). This is unlikely to greatly change the nature of the overall optimal tactic for three main reasons. Partial resistance introduces no mechanism by which applying more low-risk fungicide is detrimental. Partial resistance can lead to a case where it is better to apply more high-risk fungicide, due to convergence of the dose-response curves on the resistant and
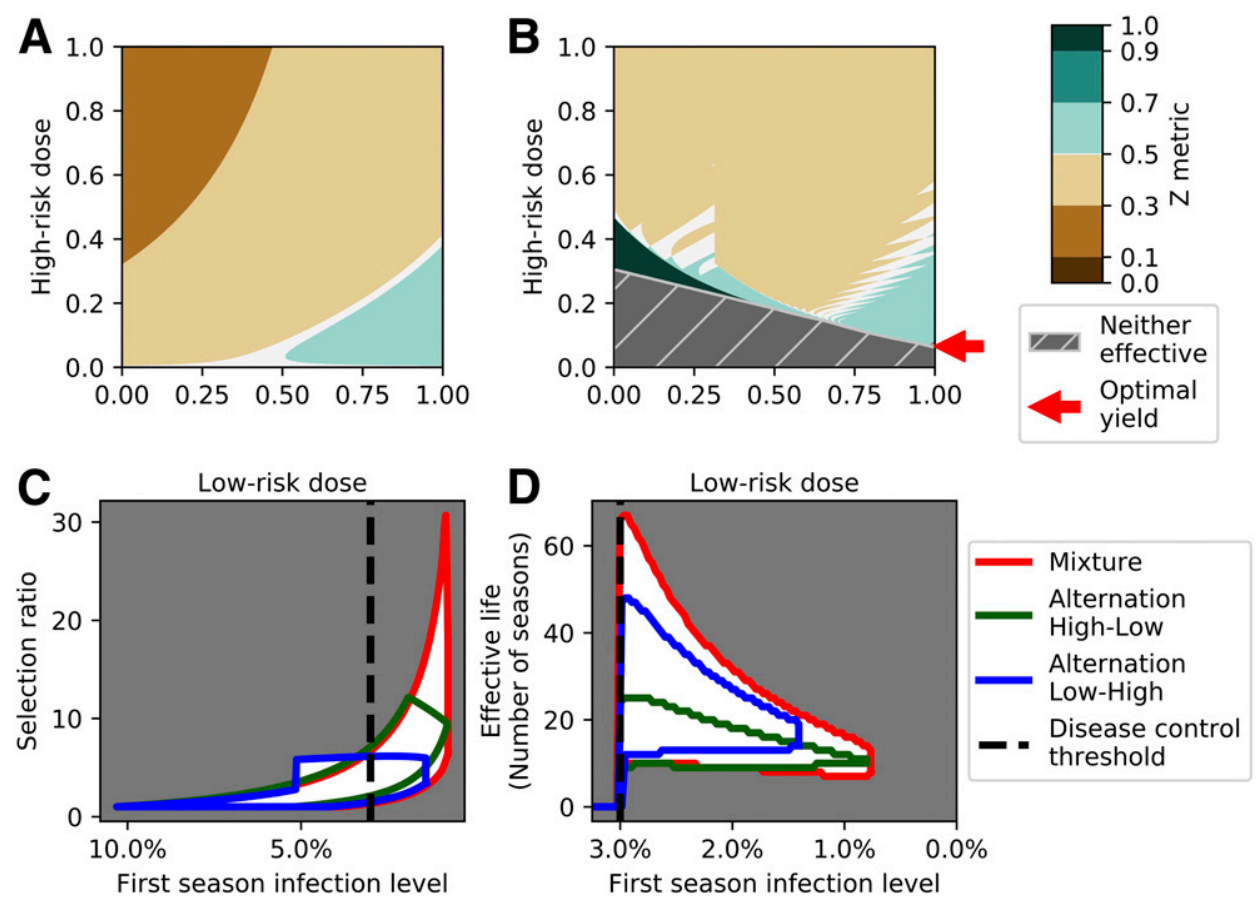

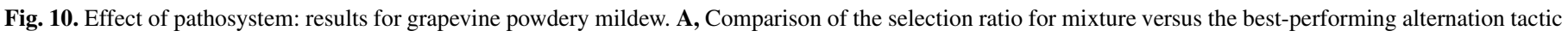

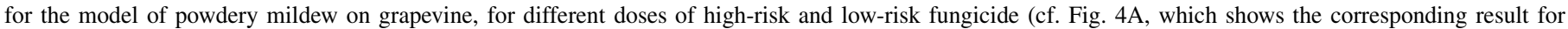

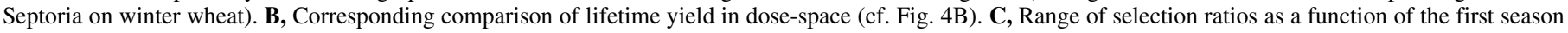

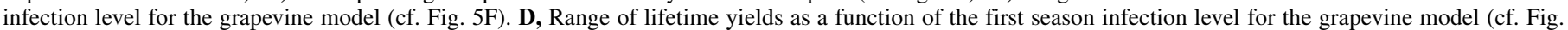

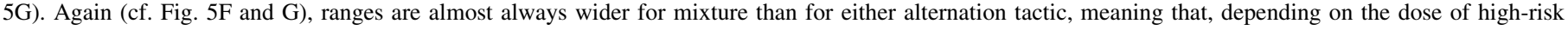

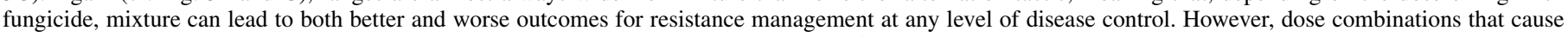

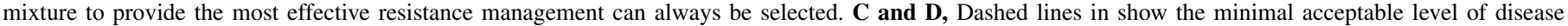
control. 
sensitive strains at higher doses (Mikaberidze et al. 2017). However, the majority of experimental evidence points to a conclusion that smaller rather than larger doses are better for resistance management (van den Bosch et al. 2011). In addition, previous modeling work, using a very similar model to our own, investigated partial resistance in the case of using two high-risk fungicides and concluded that mixture outperformed alternation (Hobbelen et al. 2013). We will return to this topic in our future work.

In this paper we have concentrated on tactics combining a highrisk with a low-risk fungicide. The current version of the standard advice for growers in the UK (FRAG-UK 2016) recommends such combinations of low-risk and high-risk fungicides for Septoria leaf blotch. In particular, Chloronitriles (chlorothalonil), Dithiocarbamates (mancozeb), and Phthalimide (folpet) are each low-risk multisite fungicides recommended by the Fungicide Resistance Action Group UK (FRAG-UK) as part of an anti-resistance management tactic. The recommendation is these low-risk products should be applied in tank mixtures with (high-risk) DMIs (demethylation inhibitors), SDHIs (succinate dehydrogenase inhibitors) or QoIs (quinone outside inhibitors), and this is strongly supported by our key result that mixtures lead to better resistance management. However, resistance management for combinations of two high-risk chemicals is potentially very important for Septoria, exemplified by the recent take up of SDHI-azole mixtures. Modeling mixtures of two high-risk fungicides will be a next step in our work. The update to the model is relatively simple, requiring only additional classes to track pathogen strains resistant to either or both fungicides. However, the recommendations of optimal tactics are likely to become more complex, since it is no longer axiomatic that one of the chemicals should always be sprayed at full dose ( $c f$. the low-risk fungicide in our work) (Hobbelen et al. 2013).

While we have identified how the optimal tactic and combination of doses could be selected, there are potentially issues in adopting our prescribed tactic. The first difficulty is that it requires the threshold between effective and ineffective control to be unambiguously identified. Given the high level of year-on-year variability typical of real disease systems (te Beest et al. 2008) and the extent to which available models do not necessarily capture the complex dynamics of epidemics accurately enough to make such a precise prediction (Gent et al. 2013), this might be rather difficult in practice. There would also be questions raised surrounding the risk aversion of growers and/or agronomists, who might, reasonably enough, wish to use higher doses of fungicides than are necessary on average to avoid failure of control in years with high disease pressures (Jørgensen et al. 2017), although in principle this might be mitigated via a sufficiently well-calibrated decision support system (Carisse et al. 2010). We have also not considered the economic aspects of our recommendations (te Beest et al. 2013), nor the potentially confounding effects of varying the timing of fungicide sprays (van den Berg et al. 2013, 2016), nor the emergence phase of resistance (Hobbelen et al. 2014; Mikaberidze et al. 2017), nor of spatial heterogeneity in coverage (Shaw 2000; Parnell et al. 2005, 2006). Nevertheless, by showing in detail and for the first time how fungicide anti-resistance tactics should properly be compared, as well as by showing how results of such comparisons can be explained using simple and intuitive epidemiological principles, our work has developed a firm base to which these complexities can be added. Our future work will do this, albeit with the expectation that using a mixture of fungicides in each spray will very often be the better tactic.

\section{APPENDIX}

Full details of the model of Septoria leaf blotch on winter wheat. The model of Septoria leaf blotch on winter wheat is a semidiscrete, compartmental model running over successive growing seasons. The model was originally derived, parameterized and tested against field data in Hobbelen et al. (2011b). It tracks the leaf area index (LAI), the area of leaf per area of ground, of different classes of leaf tissue, distinguishing a number of epidemiologically relevant compartments: the area of healthy uninfected tissue (Susceptible), the area of latent (Exposed) and infectious (Infectious) lesions, and the area of dead tissue (Removed). All classes involving the pathogen are divided into separate subcompartments for the fungicide-resistant (subscript R) and fungicide-sensitive (subscript S) strains. Note that the fungicide-resistant and fungicidesensitive strain dynamics are identical except that the high-risk fungicide does not affect the fungicide-resistant strain.

We denote the total upper leaf LAI as $A$, with

$$
A=S+E_{R}+E_{S}+I_{R}+I_{S}+R
$$

This LAI grows at rate $g$, which is monomolecular after the emergence of the first leaf tracked, and in which disease has no effect on growth

$$
g(A, t)= \begin{cases}0, & t<T_{E M E R G E} \\ r(k-A), & t \geq T_{E M E R G E}\end{cases}
$$

Living host tissues senesce at a time-dependent rate $\Gamma(t)$ governed by the time in the season relative to key growth stages

$$
\Gamma(t)= \begin{cases}0, & t<T_{G S 61} \\ 0.005\left(\frac{t-T_{G S 61}}{T_{G S 87}-T_{G S 61}}\right)+0.1 e^{-0.02\left(T_{G S 87}-t\right)}, & t \geq T_{G S 61}\end{cases}
$$

The system of differential equations describing the system is then (values and meanings of parameters are collated for easy reference in Supplementary Table S1)

$$
\begin{aligned}
& \frac{d S}{d t}=g(A, t)-\Gamma(t) S-\frac{\beta S}{A}\left(1-\varepsilon\left(C_{L}\right)\right)\left(\left(1-\varepsilon\left(C_{H}\right)\right)\left(I_{S}+P_{S}\right)+I_{R}+P_{R}\right) \\
& \frac{d E_{R}}{d t}=\frac{\beta S}{A}\left(1-\varepsilon\left(C_{L}\right)\right)\left(I_{R}+P_{R}\right)-\Gamma(t) E_{R}-\gamma E_{R} \\
& \frac{d E_{S}}{d t}=\frac{\beta S}{A}\left(1-\varepsilon\left(C_{L}\right)\right)\left(1-\varepsilon\left(C_{H}\right)\right)\left(I_{S}+P_{S}\right)-\Gamma(t) E_{S}-\gamma\left(1-\varepsilon\left(C_{H}\right)\right) E_{S} \\
& \frac{d I_{R}}{d t}=\gamma E_{R}-\mu I_{R} \\
& \frac{d I_{S}}{d t}=\gamma\left(1-\varepsilon\left(C_{H}\right)\right) E_{S}-\mu I_{S} \\
& \frac{d R}{d t}=\mu\left(I_{R}+\mathrm{I}_{S}\right)+\Gamma(t)\left(S+E_{R}+E_{S}\right) \\
& \frac{d P_{R}}{d t}=-\nu P_{R} \\
& \frac{d P_{S}}{d t}=-\nu P_{S} \\
& \frac{d C_{H}}{d t}=-\delta_{H} C_{H} \\
& \frac{d C_{L}}{d t}=-\delta_{L} C_{L}
\end{aligned}
$$

The model was slightly updated relative to the original publication (Hobbelen et al. 2011b) by modeling disease spread on the top five rather than three leaves. This avoided an edge effect whereby the spray at GS32 (very near to the start of the part of the growing season modeled in the Hobbelen paper) exerted an unrealistically large amount of selection. This was a modeling artifact due to extremely large per-capita growth rates at the start of the modeled season (caused by primary infection from the timedecaying inoculum), which was fixed by shifting the effective start of the growing season back by two phyllochrons. This change 
additionally facilitates comparison with later models which track these additional leaves (van den Berg et al. 2013). This change in the length of the models growing season required the infection rate $(\beta)$ be refitted, which was done by minimizing the squared difference between areas of infectious tissue, summed over every degree-day, between the Hobbelen et al. (2013) model and the new parameterization starting at the emergence of leaf five. The optimization was carried out for the times from the emergence of leaf three onward and with no fungicide applied.

Sensitivity analysis to model structure for the model of Septoria leaf blotch. We investigated the effect of three main features of the Septoria model: host-limited infection, whether the latent period is modeled, and phenology. Each of these features corresponds to certain features being included/omitted from the model.

- Host-limited infection. In models which do not include hostlimited infection, the infection rate is independent of the amount of host tissue, and so the terms for infection in Equation 10 are altered as follows

$$
\begin{aligned}
\text { Resistant: } & \frac{\beta S}{A}\left(1-\varepsilon\left(C_{L}\right)\right)\left(I_{R}+P_{R}\right) \rightarrow \beta\left(1-\varepsilon\left(C_{L}\right)\right)\left(I_{R}+P_{R}\right) \\
\text { Sensitive: } & \frac{\beta S}{A}\left(1-\varepsilon\left(C_{L}\right)\right)\left(1-\varepsilon\left(C_{H}\right)\right)\left(I_{S}+P_{S}\right) \\
& \rightarrow \beta\left(1-\varepsilon\left(C_{L}\right)\right)\left(1-\varepsilon\left(C_{H}\right)\right)\left(I_{S}+P_{S}\right)
\end{aligned}
$$

- Latent period. If the latent period is removed, infection moves tissue directly from the $S$ class to $I_{R}$ and $I_{S}$ without passing through $E_{R}$ and $E_{S}$.

- Phenology. If phenology is removed from the model, the senescence term $\Gamma(t)$ (Equation 9) is set to zero and the state variables corresponding to primary inoculum $\left(P_{R}\right.$ and $\left.P_{S}\right)$ are removed from the model. The epidemic is then started each season by adding a small amount of $E_{R}$ and $E_{\mathrm{S}}$ (or $I_{R}$ and $I_{S}$ if there is also no latent period in the simplified model). The amount added is the same as the amount of primary inoculum that would have been present if phenology were included in the model.

Every possible model which either includes or excludes each of these three factors is considered, leading to a total of eight different models. Since models without host-limited infection cannot provide information about the loss of green tissue to infection, they are excluded for the yield analysis, leaving four models in that case.

The infection rate parameter $(\beta)$ is refitted for each model, to allow results to be directly compared. The fitting was done by generating data for every degree-day from the full model when spraying under each of the spraying tactics (mixture and the two alternations) at four different doses of each fungicide $(0.25,0.5$, 0.75 , and full dose) and when there is no fungicide-resistant pathogen. The value of the infection rate parameter for each simplified model was chosen that minimized the sum of squared differences between the curves for the amount of $I_{S}$ over a single season for the full model and the given simplified model (Supplementary Fig. S1). Multiple doses and tactics were used in the fitting in order to give a parameter value that gave dynamics similar across the range of doses that were compared.

Full details of the model of powdery mildew on grapevine. Similar to the model of Septoria leaf blotch on winter wheat, the model of powdery mildew on grapevine is a semidiscrete, compartmental model and runs over multiple growing seasons. The model was derived and parameterized in Burie et al. (2011). It tracks healthy uninfected tissue (Susceptible), the area of latent (Exposed) and infectious (Infectious) lesions, leaf area that has developed resistance to disease due to age (Ontogenic), and dead tissue (Removed). All classes involving the pathogen are divided into a subcompartment for the fungicide-resistant (subscript R) and fungicide-sensitive (subscript $\mathrm{S}$ ) strains. Again, the fungicide-resistant and fungicide-sensitive strain dynamics are almost identical except that the high-risk fungicide does not affect the fungicide-resistant strain.

The total leaf area is

$$
A=S+E_{R}+E_{S}+I_{R}+I_{S}+R+O
$$

and this is assumed to grow logistically, with

$$
g(A, t)=r(t) A\left(1-\frac{A}{k(t)}\right)
$$

The growth parameters $r(t)$ and $k(t)$ are piecewise constant functions depending on whether $t$ is before or after shoot topping, which is the agronomic practice of removing the upper shoots to encourage secondary growth (Supplementary Table S2, in which all parameters are described and collated for easy reference). Shoot topping is modeled as occurring on day 173 of the season, and changes the value of these host growth parameters, as well as the infection rate parameter. It also leads to a $20 \%$ reduction in the size of the state variable for each compartment.

The system of differential equations describing the powdery mildew model is

$$
\begin{aligned}
& \frac{d S}{d t}=g(A, t)-\frac{\beta(t) S}{A}\left(1-\varepsilon\left(C_{L}\right)\right)\left(\left(1-\varepsilon\left(C_{H}\right)\right) I_{S}+I_{R}\right)-m S \\
& \frac{d E_{R}}{d t}=\frac{\beta(t) S}{A}\left(1-\varepsilon\left(C_{L}\right)\right) I_{R}-\gamma\left(1-\varepsilon\left(C_{L}\right)\right) E_{R} \\
& \frac{d E_{S}}{d t}=\frac{\beta(t) S}{A}\left(1-\varepsilon\left(C_{L}\right)\right)\left(1-\varepsilon\left(C_{H}\right)\right) I_{S}-\gamma\left(1-\varepsilon\left(C_{L}\right)\right)\left(1-\varepsilon\left(C_{H}\right)\right) E_{S} \\
& \frac{d I_{R}}{d t}=\gamma\left(1-\varepsilon\left(C_{L}\right)\right) E_{R}-\mu I_{R} \\
& \frac{d I_{S}}{d t}=\gamma\left(1-\varepsilon\left(C_{L}\right)\right)\left(1-\varepsilon\left(C_{H}\right)\right) E_{S}-\mu I_{S} \\
& \frac{d R}{d t}=\mu\left(I_{R}+I_{S}\right) \\
& \frac{d O}{d t}=m S \\
& \frac{d C_{H}}{d t}=-\delta_{H} C_{H} \\
& \frac{d C_{L}}{d t}=-\delta_{L} C_{L}
\end{aligned}
$$

The values for the fungicide dose-response and decay parameters were matched to data from the literature. Jyot et al. (2010) measured half-lives of around 3 days for trifloxystrobin on grapevine, and Nasr (2010) found half-lives of around 4 days for sulfur on tomatoes and squashes. Little data were available was available on fungicide effectiveness and so the assumption was made that the maximum effectiveness of both fungicides was 1 , representing the assumption that given a suitably high dose of either fungicide the growth of the pathogen can be almost entirely suppressed (even if only for a short time). Reuveni (2001) provides the reduction in disease severity when six sprays of sulfur or trifloxystrobin were used. The model was set up so that six sprays of fungicide were applied starting at day 120 , with 14 days between sprays. The values for the curvatures of both fungicides that minimized the sum of squared differences between the percentage reduction in disease severity (compared with untreated) in the model and in the 1999 dataset from Reuveni (2001) was then calculated, summing values from each individual day in the models' results.

Testing robustness of the result that mixture outperforms alternation. In order to provide further evidence that the superior performance of mixture was not specific to our chosen 
parameterization of the Septoria model, we carried out the following additional test.

- Repeat until 1,000 parameter value sets are accepted.

- Choose parameter values uniformly at random from within the ranges of all parameters displayed in Figure 7 (note this means that all parameters will in general take nondefault values).

- Check if parameters give realistic solutions and continue if so, otherwise generate new parameters. For mixtures and both alternation tactics, the reality check consists of ensuring the following.

- Yield is below 95\% when the dose of high-risk is zero and the dose of low-risk is one.

- Yield is above 95\% when a full dose of both high-risk and low-risk are applied.

- At full dose of the low-risk, find the optimal dose of high-risk and the application tactic that gives the largest lifetime yield.

As stated in the main text, no case was found that led to alternation outperforming mixture. The volume of parameter space giving realistic solutions was high, in general only requiring one or two attempts at parameter value generation to identify a reasonable set of parameters (i.e., approximately $50 \%$ of parameters tested led to a realistic parameterization of the model).

Availability of code online. An implementation of the model in the freely-available programming language Python (Python Software Foundation. Available at http://www.python.org) is online at https://doi.org/10.5281/zenodo.1230283.

\section{ACKNOWLEDGMENTS}

J. A. D. Elderfield acknowledges the Biotechnology and Biological Sciences Research Council of the United Kingdom (BBSRC) for support via a University of Cambridge DTP Ph.D. studentship. Rothamsted Research also receives support from the BBSRC. We also thank three anonymous reviewers for their helpful comments on a previous version of the manuscript.

\section{LITERATURE CITED}

Birch, C. P. D., and Shaw, M. W. 1997. When can reduced doses and pesticide mixtures delay the build-up of pesticide resistance? A mathematical model. J. Appl. Ecol. 34:1032-1042.

Brent, K. J., and Hollomon, D. W. 2007. Fungicide resistance: The assessment of risk. FRAC Monograph 2. 2nd ed. Brussels, CropLife International 52.

Burie, J., Langlais, M., and Calonnec, A. 2011. Switching from a mechanistic model to a continuous model to study at different scales the effect of vine growth on the dynamic of a powdery mildew epidemic. Ann. Bot. (Lond.) 107:885-895.

Calonnec, A., Cartolar, P., Poupot, C., Dubourdieu, D., and Darriet, P. 2004. Effects of Uncinula necator on the yield and quality of grapes (Vitis vinifera) and wine. Plant Pathol. 53:434-445.

Calonnec, A., Cartolaro, P., Delière, L., and Chadoeuf, J. 2006. Powdery mildew on grapevine: The date of primary contamination affects disease development on leaves and damage on grape. IOBC WPRS Bull. 29:67-73.

Carisse, O., Tremblay, D.-M., Jobin, T., and Walker, A. 2010. Disease decision support systems: their impact on disease management and durability of fungicide effectiveness. Pages 177-200 in: Fungicides. InTech, Rijeka, Croatia.

Cooke, L. R., Locke, T., Lockley, K. D., Phillips, A. N., Sadiq, M. D. S., and Coll, R. 2004. The effect of fungicide programs based on epoxiconazole on the control and DMI sensitivity of Rhynchosporium secalis in winter barley. Crop Prot. 23:393-406.

Cunniffe, N. J., Koskella, B., Metcalf, C. J. E., Parnell, S., Gottwald, T. R., and Gilligan, C. A. 2015. Thirteen challenges in modelling plant diseases. Epidemics 10:6-10.

Cunniffe, N. J., Stutt, R. O. J. H., van den Bosch, F., and Gilligan, C. A. 2012. Time-dependent infectivity and flexible latent and infectious periods in compartmental models of plant disease. Phytopathology 102:365-380.

Delière, L., Cartolaro, P., Léger, B., and Naud, O. 2015. Field evaluation of an expertise-based formal decision system for fungicide management of grapevine downy and powdery mildews. Pest Manag. Sci. 71:1247-1257.

Deliere, L., Miclot, A. S., Sauris, P., Rey, P., and Calonnec, A. 2010. Efficacy of fungicides with various modes of action in controlling the early stages of an Erysiphe necator-induced epidemic. Pest Manag. Sci. 66:1367-1373.
Delp, C. J. 1980. Coping with resistance to plant disease control agents. Plant Dis. 64:652-657.

Doster, M. A., Milgroom, M. G., and Fry, W. E. 1990. Quantification of factors influencing potato late blight suppression and selection for metalaxyl resistance in Phytophthora infestans: A simulation approach. Phytopathology 80:1190-1198.

Dovas, C., Skylakakis, G., and Georgopoulos, S. G. 1976. The adaptability of the benomyl-resistant population of Cercospora beticola in Northern Greece. Phytopathology 66:1452-1456.

FRAG-UK. 2016. Fungicide Resistance Management in Cereals. Fungicide Resistance Action Group (FRAG), UK.

Gadoury, D. M., Seem, R. C., Ficke, A., and Wilcox, W. F. 2003. Ontogenic resistance to powdery mildew in grape berries. Phytopathology 93:547-555

Gent, D. H., Mahaffee, W. F., McRoberts, N., and Pfender, W. F. 2013. The use and role of predictive systems in disease management. Annu. Rev. Phytopathol. 51:267-289.

Gooding, M., and Dimmock, J. 2000. Green leaf area decline of wheat flag leaves: The influence of fungicides and relationships with mean grain weight and grain yield. Ann. Appl. Biol. 136:77-84.

Gubbins, S., and Gilligan, C. A. 1999. Invasion thresholds for fungicide resistance: deterministic and stochastic analyses. Proc. Roy. Soc. B: Biol. Sci. 266:2539-2549.

Hall, R. J., Gubbins, S., and Gilligan, C. A. 2004. Invasion of drug and pesticide resistance is determined by a trade-off between treatment efficacy and relative fitness. Bull. Math. Biol. 66:825-840.

Hilker, F. M., Allen, L. J. S., Bokil, V. A., Briggs, C. J., Feng, Z., Garrett, K. A., Gross, L. J., Hamelin, F. M., Jeger, M. J., Manore, C. A., Power, A. G., Redinbaugh, M. G., Rua, M. A., and Cunniffe, N. J. 2017. Modeling virus coinfection to inform management of maize lethal necrosis in Kenya. Phytopathology 107:1095-1108.

Hobbelen, P. H. F., Paveley, N. D., Fraaije, B. A., Lucas, J. A., and van den Bosch, F. 2011b. Derivation and testing of a model to predict selection for fungicide resistance. Plant Pathol. 60:304-313.

Hobbelen, P. H. F., Paveley, N. D., Oliver, R. P., and van den Bosch, F. 2013. The usefulness of fungicide mixtures and alternation for delaying the selection for resistance in populations of Mycosphaerella graminicola on winter wheat: A modeling analysis. Phytopathology 103:690-707.

Hobbelen, P. H. F., Paveley, N. D., and van den Bosch, F. 2011a. Delaying selection for fungicide insensitivity by mixing fungicides at a low and high risk of resistance development: A modeling analysis. Phytopathology 101: 1224-1233.

Hobbelen, P. H. F., Paveley, N. D., and van den Bosch, F. 2014. The emergence of resistance to fungicides. PLoS One 9:e91910.

Jørgensen, L. N., van den Bosch, F., Oliver, R. P., Heick, T. M., and Paveley, N. D. 2017. Targeting fungicide inputs according to need. Annu. Rev. Phytopathol. 55:181-203.

Josepovits, G. 1989. A model for evaluating factors affecting the development of insensitivity to fungicides. Crop Prot. 8:106-113.

Josepovits, G., and Dobrovolszky, A. 1985. A novel mathematical approach to the prevention of fungicide resistance. Pest Manag. Sci. 16:17-22.

Jyot, G., Arora, P. K., Sahoo, S. K., Singh, B., and Battu, R. S. 2010. Persistence of trifloxystrobin and tebuconazole on grape leaves, grape berries and soil. Bull. Environ. Contam. Toxicol. 84:305-310.

Kable, P., and Jeffery, H. 1980. Selection for tolerance in organisms exposed to sprays of biocide mixtures: A theoretical model. Phytopathology 70:8-12.

Kitchen, J. L., van den Bosch, F., Paveley, N. D., Helps, J., and van den Berg, F. 2016. The evolution of fungicide resistance resulting from combinations of foliar-acting systemic seed treatments and foliar-applied fungicides: A modeling analysis. PLoS One 11:e0161887.

Lamondia, J. 2001. Management of Euonymus anthracnose and fungicide resistance in Colletotrichum gloeosporioides by alternating or mixing fungicides. J. Environ. Hortic. 19:51-55.

Mailleret, L., and Lemesle, V. 2009. A note on semi-discrete modelling in the life sciences. Philos. Trans. Series A, Mathematical, Physical, and Engineering Sciences 367:4779-4799.

Mammeri, Y., Burie, J. B., Langlais, M., and Calonnec, A. 2014. How changes in the dynamic of crop susceptibility and cultural practices can be used to better control the spread of a fungal pathogen at the plot scale? Ecol. Modell. 290:178-191.

Mikaberidze, A., Mcdonald, B. A., and Bonhoeffer, S. 2014. Can high-risk fungicides be used in mixtures without selecting for fungicide resistance? Phytopathology 104:324-331.

Mikaberidze, A., Paveley, N., Bonhoeffer, S., and van den Bosch, F. 2017. Emergence of resistance to fungicides: The role of fungicide dose. Phytopathology 107:545-560.

Milgroom, M., and Fry, W. 1988. A simulation analysis of the epidemiological principles for fungicide resistance management in pathogen populations. Phytopathology 78:565-570. 
Nasr, I. N. 2010. Gas chromatographic determination of sulfur residues in tomatoes and squash fruits. J. Appl. Sci. Res. 6:1474-1476.

Parnell, S., Gilligan, C. A., and van den Bosch, F. 2005. Small-scale fungicide spray heterogeneity and the coexistence of resistant and sensitive pathogen strains. Phytopathology 95:632-639.

Parnell, S., van den Bosch, F., and Gilligan, C. A. 2006. Large-scale fungicide spray heterogeneity and the regional spread of resistant pathogen strains. Phytopathology 96:549-555.

Paveley, N., Blake, J., Gladders, P., and Cockerell, V. 2014. The Wheat Disease Management Guide. Home-Grown Cereals Authority, United Kingdom.

Pool, R. M., Pearson, R. C., Welser, M. J., Lakso, A. N., and Seem, R. C. 1984. Influence of powdery mildew on yield and growth of Rosette grapevines. Plant Dis. 68:590-593.

Reuveni, M. 2001. Activity of trifloxystrobin against powdery and downy mildew diseases of grapevines. Can. J. Plant Pathol. 23:52-59.

Russell, P. E. 2005. A century of fungicide evolution. J. Agric. Sci. 143:11-25.

Sanders, P. L., Houser, W. L., Parish, P. J., and Cole, H. 1985. Reduced-rate fungicide mixtures to delay fungicide resistance and to control selected turfgrass disease. Plant Dis. 69:939-943.

Savary, S., Delbac, L., Rochas, A., Taisant, G., and Willocquet, L. 2009. Analysis of nonlinear relationships in dual epidemics, and its application to the management of grapevine downy and powdery mildews. Phytopathology 99:930-942.

Shaw, M. 1989a. Independent action of fungicides and its consequences for strategies to retard the evolution of fungicide resistance. Crop Prot. 8:405-411.

Shaw, M. 1989b. A model of the evolution of polygenically controlled fungicide resistance. Plant Pathol. 38:44-55.

Shaw, M. W. 2000. Models of the effects of dose heterogeneity and escape on selection pressure for pesticide resistance. Phytopathology 90:333-339.

Shaw, M. W. 2006. Is there such a thing as a fungicide resistance strategy? A modeler's perspective. Asp. Appl. Biol. 78:95-103.

Skylakakis, G. 1981. Effects of alternating and mixing pesticides on the buildup of fungal resistance. Phytopathology 71:1119-1121.
Staub, T., and Sozzi, D. 1983. Recent practical experiences with fungicide resistance. Pages 591-598 in: Proc. 10th Int. Congress Plant Protection.

te Beest, D. E., Paveley, N. D., Shaw, M. W., and van den Bosch, F. 2008. Disease-weather relationships for powdery mildew and yellow rust on winter wheat. Phytopathology 98:609-617.

te Beest, D. E., Paveley, N. D., Shaw, M. W., and van den Bosch, F. 2013. Accounting for the economic risk caused by variation in disease severity in fungicide dose decisions, exemplified for Mycosphaerella graminicola on winter wheat. Phytopathology 103:666-672.

Vali, R. J., and Moorman, G. W. 1992. Influence of selected fungicide regimes on frequency of dicarboximide-resistant and dicarboximide-sensitive strains of Botrytis cinerea. Plant Dis. 76:919-924.

van den Berg, F., Paveley, N. D., and van den Bosch, F. 2016. Dose and number of applications that maximize fungicide effective life exemplified by Zymoseptoria tritici on wheat-A model analysis. Plant Pathol. 65: 1380-1389.

van den Berg, F., van den Bosch, F., and Paveley, N. D. 2013. Optimal fungicide application timings for disease control are also an effective antiresistance strategy: A case study for Zymoseptoria tritici (Mycosphaerella graminicola) on wheat. Phytopathology 103:1209-1219.

van den Bosch, F., and Gilligan, C. A. 2008. Models of fungicide resistance dynamics. Annu. Rev. Phytopathol. 46:123-147.

van den Bosch, F., Oliver, R., van den Berg, F., and Paveley, N. 2014a. Governing principles can guide fungicide-resistance management tactics. Annu. Rev. Phytopathol. 52:175-195.

van den Bosch, F., Paveley, N., Shaw, M., Hobbelen, P., and Oliver, R. 2011. The dose rate debate: does the risk of fungicide resistance increase or decrease with dose? Plant Pathol. 60:597-606.

van den Bosch, F., Paveley, N., van den Berg, F., Hobbelen, P., and Oliver, R. 2014b. Mixtures as a fungicide resistance management tactic. Phytopathology 104:1264-1273.

Waggoner, P., and Berger, R. 1987. Defoliation, disease, and growth. Phytopathology 77:393-398. 